\title{
The existence of infinitely many solutions for nonlinear elliptic equations involving $p$-Laplace type operators in $\mathbb{R}^{N}$
}

\author{
Yun-Ho Kimª , Jung-Hyun Bae ${ }^{b, *}$, Jongrak Lee ${ }^{c}$ \\ ${ }^{a}$ Department of Mathematics Education, Sangmyung University, Seoul 03016, Republic of Korea. \\ ${ }^{b}$ Department of Mathematics, Sungkyunkwan University, Suwon 16419, Republic of Korea. \\ ${ }^{c}$ Institute of Mathematical Sciences, Ewha Womans University, Seoul 03760, Republic of Korea. \\ Communicated by Y. J. Cho
}

\begin{abstract}
We are concerned with the following nonlinear elliptic equations

$$
-\operatorname{div}(\varphi(x, \nabla u))+b(x)|u|^{p-2} u=\lambda f(x, u) \text { in } \mathbb{R}^{N},
$$

where the function $\varphi(x, v)$ is of type $|v|^{p-2} v, b: \mathbb{R}^{N} \rightarrow(0, \infty)$ is a continuous potential function, $\lambda$ is a real parameter, and $f: \mathbb{R}^{N} \times \mathbb{R} \rightarrow \mathbb{R}$ is a Carathéodory function. In this paper, under suitable assumptions, we show the existence of infinitely many weak solutions for the problem above without assuming the Ambrosetti and Rabinowitz condition, by using the fountain theorem. Next, we give a result on the existence of a sequence of solutions for the problem above converging to zero in the $\mathrm{L}^{\infty}$-norm by employing the Moser iteration under appropriate conditions. (C)2017 All rights reserved.
\end{abstract}

Keywords: p-Laplace type, weak solution, iteration method, fountain theorem.

2010 MSC: 35D30, 35J20, 35J60, 35J92.

\section{Introduction}

The celebrated mountain pass theorem of Ambrosetti and Rabinowitz [2] has become one of main tools for solving elliptic equations of variational type. Ambrosetti and Rabinowitz [2] proved the existence of infinitely many distinct pairs of solutions of second order uniformly elliptic equations, subject to the Dirichlet boundary condition as applications of their mountain pass theorem. Due to a generalization of the mountain pass theorem in [2], the existence of infinitely many solutions of the semilinear elliptic Dirichlet boundary value problem was investigated by Bartsch [3].

The multiplicity result of solutions for the following semilinear elliptic problem

$$
-\Delta u+b(x) u=\lambda f(x, u) \text { in } \mathbb{R}^{N}
$$

\footnotetext{
*Corresponding author

Email addresses: kyh1213@smu.ac.kr (Yun-Ho Kim), hoi1000sa@skku.edu (Jung-Hyun Bae), jrlee0124@ewha.ac.kr (Jongrak Lee)
}

doi:10.22436/jnsa.010.04.67

Received 2016-12-19 
was studied by Bartsch and Wang [5], as applying the fountain theorem which was first introduced in [4, Theorem 2.25] (see also [34]). It is well-known that the main difficulty in treating the problem (1.1) in $\mathbb{R}^{N}$ arises from the lack of the compactness of the Sobolev embedding, which prevents from checking directly that the energy functional associated with (1.1) satisfies the Palais-Smale condition. To overcome the difficulty of the noncompact embedding, they established a new compact embedding result under suitable conditions on the potential $b(x)$. To this end, as showing the compactness of the Palais-Smale sequence for an energy functional which plays an important role in applying the fountain theorem, they proved the existence of infinitely many solutions for the problem (1.1). Motivated by the work of [5], many authors considered elliptic problems involving the $p$-Laplacian in the whole space. We refer the readers to [22, 26]; see also [1,24] for the case of variable exponents.

In the present paper, we deal with the following nonlinear elliptic equations of the p-Laplace type

$$
-\operatorname{div}(\varphi(x, \nabla u))+b(x)|u|^{p-2} u=\lambda f(x, u) \text { in } \mathbb{R}^{N}
$$

where the function $\varphi(x, v)$ is of type $|v|^{p-2} v, b: \mathbb{R}^{N} \rightarrow(0, \infty)$ is a continuous potential function, $\lambda$ is a real parameter, and $f: \mathbb{R}^{N} \times \mathbb{R} \rightarrow \mathbb{R}$ is a Carathéodory function. As a natural extension of the $p$-Laplacian operator, many researchers have been widely studied nonlinear elliptic equations of the $p$-Laplace type operator in various ways; see $[8,10,11,22,23]$ and references therein.

The first aim of this paper is to demonstrate the existence of an unbounded sequence of solutions for the problem (1.2) without assuming the following Ambrosetti and Rabinowitz condition ((AR)-condition for short) which is imposed on the nonlinearity $f(x, t)$ :

(AR) There exist positive constants $N$ and $\zeta$ such that $\zeta>p$ and

$$
0<\zeta \mathrm{F}(x, t) \leqslant f(x, t) t, \quad \text { for } x \in \Omega \text { and }|t| \geqslant N,
$$

where $F(x, t)=\int_{0}^{t} f(x, s) d s$, and $\Omega$ is a bounded domain in $\mathbb{R}^{N}$.

The main role of (AR)-condition in applying critical point theory is to ensure the boundedness of the Palais-Smale sequence of the functional with respect to the problem (1.2). However, this condition is very restrictive, and thus undoubtedly eliminates many nonlinearities. Accordingly, many authors in $[18,25,27-29,36]$ have made an effort to drop (AR)-condition in recent years. In particular, Jeanjean [17] obtained the existence and multiplicity of solutions for the Laplacian equation on the whole space $\mathbb{R}^{N}$ assuming the following condition (Je) below:

(Je) There exists $\eta \geqslant 1$ such that

$$
\eta \mathcal{F}(x, t) \geqslant \mathcal{F}(x, s t),
$$

for all $(x, t) \in \mathbb{R}^{N} \times \mathbb{R}$ and $s \in[0,1]$, where $\mathcal{F}(x, t)=f(x, t) t-p F(x, t)$.

This condition (Je) is weaker than (AR)-condition and in past years there are numerous studies dealing with the $p$-Laplacian problem by assuming (Je); see $[7,27,28]$. We refer to $[1,31]$ for the case of variable exponents and [32] for the fractional p-Laplacian. From this point of view, we discuss the existence of an unbounded sequence of solutions for the problem (1.2) without assuming (AR)-condition under a suitable assumption for $f$ which is slightly different from (Je). The method of approach is to apply the fountain theorem given in [34, Theorem 3.6].

The second aim is to prove the existence of small solutions for the problem (1.2), which means that the sequence of solutions converges to 0 in the $L^{\infty}$-norm, relies only on local behavior under the conditions on $f(x, u)$ only for sufficiently small $u$. To do this, we use a method by means of global variational formulations introduced by Wang [33]. He obtained the existence of infinitely many solutions for nonlinear boundary value problems which is rather a local phenomenon by modifying and extending the functional $f(x, u)$. Utilizing the argument in [33], Naimen [30] showed that nonlinear Neumann problems have infinitely many solutions whose $\mathrm{L}^{\infty}$-norms converge to zero. In this direction, many authors considered the results for the nonlinear equations on a bounded domain in $\mathbb{R}^{N}$; see $[9,15,19,31]$. To the best of 
our knowledge, such a result on the whole space $\mathbb{R}^{N}$ is very rare. The strategy to obtain this multiplicity result involves the following two steps: Firstly, we give a regularity type result on the $L^{\infty}$-bound of solutions for the problem (1.2) by applying the Moser iteration method based on the work of Drábek, Kufner, and Nicolosi in [12]. It is worth noticing that the problem (1.2) includes the potential term, so more complicated analysis has to be carefully carried out in comparison with nonlinear equations given in [12]. Secondly, we establish the existence of a sequence of solutions which converges to zero in the $\mathrm{L}^{\infty}$-norm with the aid of the regularity result.

This paper is organized as follows: In Section 2, under certain conditions on $\varphi$ and $f$, we establish the existence of an unbounded solutions for the problem (1.2) by employing the fountain theorem (see Theorem 2.11). In Section 3, we give a result on the existence of a sequence of solutions for the problem (1.2) converging to zero in the $L^{\infty}$-norm via the Moser iteration under the appropriate conditions (see Proposition 3.1 and Theorem 3.5).

\section{Existence of solutions}

In this section, we shall give the proof of the existence of nontrivial weak solutions for the problem (1.2), by applying the mountain pass theorem and the fountain theorem.

Let $1<p<N$ and $p^{*}:=N p /(N-p)$ denotes the Sobolev conjugate of $p$. Let the potential $b \in$ $\mathrm{C}\left(\mathbb{R}^{N}, \mathbb{R}\right)$ be continuous. Suppose that

(B) $b \in C\left(\mathbb{R}^{N}, \mathbb{R}\right), \inf _{x \in \mathbb{R}^{N}} b(x) \geqslant b_{0}>0$, meas $\left\{x \in \mathbb{R}^{N}:-\infty<b(x) \leqslant M\right\}<+\infty, \quad \forall M \in \mathbb{R}$.

Define the linear subspace

$$
X:=\left\{u \in W^{1, p}\left(\mathbb{R}^{N}\right): \int_{\mathbb{R}^{N}}|\nabla u|^{p} d x+\int_{\mathbb{R}^{N}} b(x)|u|^{p} d x<\infty\right\},
$$

with $b$ satisfying the condition (B), then $X$ is a reflexive separable Banach space with the norm

$$
\|u\|_{X}=\left(\int_{\mathbb{R}^{N}}|\nabla u|^{p} d x+\int_{\mathbb{R}^{N}} b(x)|u|^{p} d x\right)^{\frac{1}{p}},
$$

which is equivalent to the norm $\|\cdot\|_{W^{1, p}\left(\mathbb{R}^{N}\right)}$ given by

$$
\|u\|_{W^{1, p}\left(\mathbb{R}^{N}\right)}=\left(\int_{\mathbb{R}^{N}}|\nabla u|^{p} \mathrm{~d} x+\int_{\mathbb{R}^{N}}|u|^{p} \mathrm{~d} x\right)^{\frac{1}{\mathfrak{p}}} .
$$

Lemma 2.1. The following statements hold:

(i) There is a continuous embedding $\mathrm{W}^{1, \mathrm{p}}\left(\mathbb{R}^{\mathrm{N}}\right) \hookrightarrow \mathrm{L}^{\mathrm{s}}\left(\mathbb{R}^{\mathrm{N}}\right)$ for any $\mathrm{s} \in\left[\mathrm{p}, \mathrm{p}^{*}\right]$.

(ii) If $\mathrm{b}$ satisfies the assumption (B), then there is a compact embedding $\mathrm{X} \hookrightarrow \mathrm{L}^{\mathrm{s}}\left(\mathbb{R}^{\mathrm{N}}\right)$ for any $\mathrm{s} \in\left[\mathrm{p}, \mathrm{p}^{*}\right)$.

Throughout this paper, let $X$ be the completion of $C_{0}^{\infty}\left(\mathbb{R}^{N}\right)$ and let $X^{*}$ be the dual space of $X$. Furthermore, $\langle\cdot, \cdot\rangle$ denotes the pairing of $X$ and its dual $X^{*}$ and Euclidean scalar product on $\mathbb{R}^{\mathrm{N}}$, respectively.

Definition 2.2. We say that $u \in X$ is a weak solution of the problem (1.2), if

$$
\int_{\mathbb{R}^{N}} \varphi(x, \nabla u) \cdot \nabla v d x+\int_{\mathbb{R}^{N}} b(x)|u|^{p-2} u v d x=\lambda \int_{\mathbb{R}^{N}} f(x, u) v d x
$$

for all $v \in X$. 
We assume that $\varphi: \mathbb{R}^{N} \times \mathbb{R}^{N} \rightarrow \mathbb{R}^{N}$ is a continuous function with the continuous derivative with respect to $v$ of the mapping $\Phi_{0}: \mathbb{R}^{N} \times \mathbb{R}^{N} \rightarrow \mathbb{R}, \Phi_{0}=\Phi_{0}(x, v)$, that is, $\varphi(x, v)=\frac{\mathrm{d}}{\mathrm{d} v} \Phi_{0}(x, v)$. Suppose that $\varphi$ and $\Phi_{0}$ satisfy the following assumptions:

(J1) The equality

$$
\Phi_{0}(x, \mathbf{0})=0,
$$

holds for almost all $x \in \mathbb{R}^{N}$.

(J2) There are a function $a \in \mathrm{L}^{\mathrm{p}^{\prime}}\left(\mathbb{R}^{\mathrm{N}}\right)$ and a nonnegative constant $\mathrm{a}_{0}$ such that

$$
|\varphi(x, v)| \leqslant a(x)+a_{0}|v|^{p-1},
$$

holds for almost all $x \in \mathbb{R}^{N}$ and for all $v \in \mathbb{R}^{N}$, where $1 / p+1 / p^{\prime}=1$.

(J3) $\Phi_{0}(x, \cdot)$ is strictly convex in $\mathbb{R}^{N}$ for all $x \in \mathbb{R}^{N}$.

(J4) The relations

$$
\mathrm{d}|v|^{\mathrm{p}} \leqslant \varphi(x, v) \cdot v \quad \text { and } \quad \mathrm{d}|v|^{\mathrm{p}} \leqslant p \Phi_{0}(x, v),
$$

hold for all $x \in \mathbb{R}^{N}$ and $v \in \mathbb{R}^{N}$, where $d$ is a positive constant.

(J5) There exists a constant $\theta \geqslant p$ such that

$$
H(x, s v) \leqslant H(x, v),
$$

for $v \in \mathbb{R}^{N}$ and $s \in[0,1]$, where $H(x, v)=\theta \Phi_{0}(x, v)-\varphi(x, v) \cdot v$, for all $x \in \mathbb{R}^{N}$.

Let us define the functional $\Phi: X \rightarrow \mathbb{R}$ by

$$
\Phi(\mathfrak{u})=\int_{\mathbb{R}^{\mathrm{N}}} \Phi_{0}(x, \nabla \mathfrak{u}) \mathrm{d} x+\frac{1}{\mathrm{p}} \int_{\mathbb{R}^{\mathrm{N}}} \mathrm{b}(\mathrm{x})|\mathfrak{u}|^{\mathrm{p}} \mathrm{d} \mathrm{x} .
$$

Under the assumptions (J1), (J2), and (J4), it follows from [24, Lemma 3.2] that the functional $\Phi$ is welldefined on $X, \Phi \in C^{1}(X, \mathbb{R})$, and its Fréchet derivative is given by

$$
\left\langle\Phi^{\prime}(u), v\right\rangle=\int_{\mathbb{R}^{N}} \varphi(x, \nabla u) \cdot \nabla v d x+\int_{\mathbb{R}^{N}} \mathfrak{b}(x)|u|^{p-2} u v d x .
$$

Let us set $F(x, t)=\int_{0}^{t} f(x, s) d s$. For $1<p<q<p^{*}$, we assume that

(F1) $f: \mathbb{R}^{N} \times \mathbb{R} \rightarrow \mathbb{R}$ satisfies the Carathéodory condition in the sense that $f(\cdot, t)$ is measurable for all $t \in \mathbb{R}$ and $f(x, \cdot)$ is continuous for almost all $x \in \mathbb{R}^{N}$.

(F2) f satisfies the following growth condition: for all $(x, t) \in \mathbb{R}^{N} \times \mathbb{R}$,

$$
|f(x, t)| \leqslant \sigma(x)|t|^{q-1},
$$

where $\sigma \in \mathrm{L}^{\gamma_{1}}\left(\mathbb{R}^{\mathrm{N}}\right) \cap \mathrm{L}^{\infty}\left(\mathbb{R}^{\mathrm{N}}\right)$ with $\gamma_{1}=\frac{\mathrm{p}^{*}}{\mathrm{p}^{*}-\mathrm{q}}$.

(F3) $\lim _{|t| \rightarrow \infty} \frac{F(x, t)}{|t|^{p}}=\infty$ uniformly for almost all $x \in \mathbb{R}^{N}$.

(F4) There exists a constant $\mu \geqslant 1$ such that

$$
\mu \mathcal{F}(x, t) \geqslant \mathcal{F}(x, s t),
$$

for $(x, t) \in \mathbb{R}^{N} \times \mathbb{R}$ and $s \in[0,1]$, where $\mathcal{F}(x, t)=f(x, t) t-\theta F(x, t)$ and $\theta$ is given in (J5).

(F5) $f(x,-t)=-f(x, t)$ holds for all $(x, t) \in \mathbb{R}^{N} \times \mathbb{R}$.

Define the functional $\Psi: X \rightarrow \mathbb{R}$ by

$$
\Psi(u)=\int_{\mathbb{R}^{N}} F(x, u) d x
$$


Then it is easy to check that $\Psi \in C^{1}(X, \mathbb{R})$ and its Fréchet derivative is

$$
\left\langle\Psi^{\prime}(u), v\right\rangle=\int_{\mathbb{R}^{N}} f(x, u) v d x,
$$

for any $u, v \in X$ (see [34]). Next we define the functional $\mathrm{I}_{\lambda}: \mathrm{X} \rightarrow \mathbb{R}$ by

$$
\mathrm{I}_{\lambda}(\mathrm{u})=\Phi(\mathrm{u})-\lambda \Psi(\mathrm{u}) .
$$

Then it follows that the functional $\mathrm{I}_{\lambda} \in \mathrm{C}^{1}(X, \mathbb{R})$ and its Fréchet derivative is

$$
\left\langle\mathrm{I}_{\lambda}^{\prime}(\mathrm{u}), v\right\rangle=\int_{\mathbb{R}^{N}} \varphi(x, \nabla u) \cdot \nabla v d x+\int_{\mathbb{R}^{N}} \mathrm{~b}(x)|u|^{p-2} u v d x-\lambda \int_{\mathbb{R}^{N}} f(x, u) v d x,
$$

for any $u, v \in X$.

Since the assumption (F4) is weaker than the following assumption that

$$
\frac{f(x, t)}{|t|^{\theta-2} t} \text { is increasing in } t>0 \text { and decreasing in } t<0,
$$

for any $x \in \mathbb{R}^{N}$, we check that the following examples satisfy the assumption (F4) by applying the assumption (2.2).

Example 2.3. Let us consider

$$
\varphi(x, v)=|v|^{p-2} v, \quad \text { and } \quad f(x, t)=|t|^{q-2} t \log (1+|t|),
$$

for all $v \in \mathbb{R}^{N}$ and for all $t \in \mathbb{R}$. It is clear that the function $\varphi$ satisfies the assumptions (J1)-(J5) when $\theta=p$. Also, it is obvious that the function $f$ satisfies the conditions (F1)-(F3) hold. Since the relation

$$
\frac{f(x, t)}{|t|^{p-2} t}=\frac{|t|^{q-2} t \log (1+|t|)}{|t|^{p-2} t}=|t|^{q-p} \log (1+|t|)
$$

is increasing in $t>0$ and decreasing in $t<0$, if $q>p=\theta$ for all $x \in \mathbb{R}^{N}$, it follows that the assumption (F4) holds.

Example 2.4. Let us consider

$$
\varphi(x, v)=\left(1+\frac{|v|^{p}}{\sqrt{1+|v|^{2 p}}}\right)|v|^{p-2} v, \quad \text { and } \quad f(x, t)=|t|^{q-2} t \log (1+|t|),
$$

for all $v \in \mathbb{R}^{N}$ and for all $t \in \mathbb{R}$. In this case, put

$$
\Phi_{0}(x, v)=\frac{1}{p}\left(|v|^{p}+\sqrt{1+|v|^{2 p}}-1\right)
$$

for all $v \in \mathbb{R}^{N}$. It is immediately apparent that the conditions (J1)-(J4) hold. A similar argument in [6] implies that the assumption (J5) holds when $\theta=2 p$. For the sake of convenience, we give the detailed proof. In fact, we have, for all $s \in[0,1]$

$$
\begin{aligned}
H(x, v)-H(x, s v) & =2 p \Phi_{0}(x, v)-\varphi(x, v) \cdot v-2 p \Phi_{0}(x, s v)+\varphi(x, s v) \cdot s v \\
& =2 p\left(\frac{1}{p}|v|^{p}+\frac{\sqrt{1+|v|^{2 p}}}{p}-\frac{1}{p}|s v|^{p}-\frac{\sqrt{1+|s v|^{2 p}}}{p}\right)
\end{aligned}
$$




$$
\begin{aligned}
& -\left(1+\frac{|v|^{p}}{\sqrt{1+|v|^{2 p}}}\right)|v|^{p}+\left(1+\frac{|s v|^{p}}{\sqrt{1+|s v|^{2 p}}}\right)|s v|^{p} \\
= & |v|^{p}-|s v|^{p} \\
& +2 \sqrt{1+|v|^{2 p}}-\frac{|v|^{2 p}}{\sqrt{1+|v|^{2 p}}}-2 \sqrt{1+|s v|^{2 p}}+\frac{|s v|^{2 p}}{\sqrt{1+|s v|^{2 p}}} \\
\geqslant & 2 \sqrt{1+|v|^{2 p}}-\frac{|v|^{2 p}}{\sqrt{1+|v|^{2 p}}}-2 \sqrt{1+|s v|^{2 p}}+\frac{|s v|^{2 p}}{\sqrt{1+|s v|^{2 p}}} .
\end{aligned}
$$

Define the functional $h: \mathbb{R}^{N} \times\left(\mathbb{R}^{+} \cup\{0\}\right) \rightarrow \mathbb{R}$ by

$$
h(x, \ell)=2 \sqrt{1+\ell^{2 p}}-\frac{\ell^{2 p}}{\sqrt{1+\ell^{2 p}}} .
$$

Then we obtain

$$
\frac{\partial}{\partial \ell} h(x, \ell)=\left(2 p \ell^{2 p-1}-2 p \ell^{2 p-1}\right) \frac{1}{\sqrt{1+\ell^{2 p}}}+\frac{p \ell^{4 p-1}}{\left(1+\ell^{2 p}\right) \sqrt{1+\ell^{2 p}}} \geqslant 0,
$$

for all $(x, \ell) \in \mathbb{R}^{N} \times\left(\mathbb{R}^{+} \cup\{0\}\right)$. This implies that

$$
H(x, v)-H(x, s v) \geqslant 0 .
$$

Also the same argument as in the previous example shows that $f$ satisfies the assumption (F4) if we set $q>2 p=\theta$ for all $x \in \mathbb{R}^{N}$.

Next, taking inspiration from the argument given in [21, Theorem 4.1], we will show that the operator $\Phi^{\prime}$ is of type $\left(S_{+}\right)$when $X$ is the Sobolev space on the whole space $\mathbb{R}^{N}$; see also $[8,24]$. For bounded domains in $\mathbb{R}^{N}$, we refer to $[10,21]$.

Lemma 2.5. Assume that (B) and (J1)-(J4) hold. Then the functional $\Phi: X \rightarrow \mathbb{R}$ is convex and weakly lower semicontinuous on X. Moreover, the operator $\Phi^{\prime}$ is of type $\left(S_{+}\right)$, i.e., if $u_{n} \rightarrow u$ in $X$ and

$$
\limsup _{n \rightarrow \infty}\left\langle\Phi^{\prime}\left(u_{n}\right)-\Phi^{\prime}(u), u_{n}-u\right\rangle \leqslant 0,
$$

then $u_{n} \rightarrow u$ in $X$ as $n \rightarrow \infty$.

Proof. To show that $\Phi^{\prime}$ is of type $\left(S_{+}\right)$, let $\left\{u_{n}\right\}$ be a sequence in $X$ such that $u_{n} \rightarrow u$ in $X$ as $n \rightarrow \infty$ and

$$
\limsup _{n \rightarrow \infty}\left\langle\Phi^{\prime}\left(u_{n}\right)-\Phi^{\prime}(u), u_{n}-u\right\rangle \leqslant 0
$$

Since $\Phi$ is strictly convex by the assumption (J3), it is obvious that the operator $\Phi^{\prime}$ is monotone, that is,

$$
\left\langle\Phi^{\prime}\left(u_{n}\right)-\Phi^{\prime}(u), u_{n}-u\right\rangle \geqslant 0 .
$$

By (2.3) and (2.4), we have

$$
\begin{aligned}
\lim _{n \rightarrow \infty} \int_{\mathbb{R}^{N}}\left(\varphi\left(x, \nabla u_{n}\right)-\varphi(x, \nabla u)\right) \cdot\left(\nabla u_{n}-\nabla u\right) d x \\
+\lim _{n \rightarrow \infty} \int_{\mathbb{R}^{N}} b(x)\left(\left|u_{n}\right|^{p-2} u_{n}-|u|^{p-2} u\right)\left(u_{n}-u\right) d x \\
\quad=\lim _{n \rightarrow \infty}\left\langle\Phi^{\prime}\left(u_{n}\right)-\Phi^{\prime}(u), u_{n}-u\right\rangle=0,
\end{aligned}
$$


which yields that

$$
\lim _{n \rightarrow \infty} \int_{\mathbb{R}^{N}}\left(\varphi\left(x, \nabla u_{n}\right)-\varphi(x, \nabla u)\right) \cdot\left(\nabla u_{n}-\nabla u\right) d x=0
$$

Now, we prove that the sequence $\left\{\left|\nabla u_{n}\right|^{p}\right\}$ is uniformly integrable in $\mathbb{R}^{N}$, i.e., for each $\varepsilon>0$, there exists $\delta>0$ such that if $\mathrm{H}$ is a measurable subset of $\mathbb{R}^{\mathrm{N}}$ with meas $(\mathrm{H}) \leqslant \delta$ (meas $(\mathrm{H})$ is the Lebesgue measure of $H$ ), then

$$
\int_{H}\left|\nabla u_{n}\right|^{p} d x \leqslant \varepsilon
$$

for every $n \in \mathbb{N}$. Given $\varepsilon>0$, the equation (2.5) implies that there exists $n_{1} \in \mathbb{N}$ such that for $n \geqslant n_{1}$, we get

$$
\int_{\mathrm{H}}\left(\varphi\left(x, \nabla \mathbf{u}_{\mathfrak{n}}\right)-\varphi(x, \nabla \mathfrak{u})\right) \cdot\left(\nabla \mathbf{u}_{\mathfrak{n}}-\nabla \mathfrak{u}\right) \mathrm{d} x \leqslant \frac{\varepsilon d}{8}
$$

for any measurable subset $H$ of $\mathbb{R}^{N}$, which says

$$
\int_{H} \varphi\left(x, \nabla u_{n}\right) \cdot \nabla u_{n} d x \leqslant \frac{\varepsilon d}{8}+\int_{H} \varphi(x, \nabla u) \cdot \nabla u_{n}+\varphi\left(x, \nabla u_{n}\right) \cdot \nabla u-\varphi(x, \nabla u) \cdot \nabla u d x .
$$

According to the assumption (J4) we obtain that

$$
\int_{H} \varphi\left(x, \nabla u_{n}\right) \cdot \nabla u_{n} d x \geqslant \int_{H} d\left|\nabla u_{n}\right|^{p} d x .
$$

Given $\varepsilon_{0}>0$, there exists a constant $C\left(\varepsilon_{0}\right)>0$ depending on $\varepsilon_{0}$ such that for all $\mathrm{a}, \mathrm{b} \in \mathbb{R}$,

$$
a b \leqslant \varepsilon_{0}|a|^{p}+C\left(\varepsilon_{0}\right)|b|^{p^{\prime}} .
$$

By using this inequality, (J2), and (J4), we deduce that

$$
\begin{aligned}
\varphi(x, \nabla u) \cdot \nabla u_{n}+ & \varphi\left(x, \nabla u_{n}\right) \cdot \nabla u-\varphi(x, \nabla u) \cdot \nabla u \\
\leqslant & |a(x)| \nabla u_{n}+a_{0}|\nabla u|^{p-1} \nabla u_{n}+|a(x)| \nabla u+a_{0}\left|\nabla u_{n}\right|^{p-1} \nabla u-d|\nabla u|^{p} \\
\leqslant & C\left(\frac{d}{4}\right)|a(x)|^{p^{\prime}}+\frac{d}{4}\left|\nabla u_{n}\right|^{p}+a_{0} C\left(\frac{d}{4 a_{0}}\right)|\nabla u|^{p}+\frac{d}{4}\left|\nabla u_{n}\right|^{p} \\
& +\frac{d}{4 a_{0}}|a(x)|^{p^{\prime}}+C\left(\frac{d}{4 a_{0}}\right)|\nabla u|^{p}+\frac{d}{4}\left|\nabla u_{n}\right|^{p}+a_{0} C\left(\frac{d}{4 a_{0}}\right)|\nabla u|^{p} \\
= & \left(C\left(\frac{d}{4}\right)+\frac{d}{4 a_{0}}\right)|a(x)|^{p^{\prime}}+\frac{3 d}{4}\left|\nabla u_{n}\right|^{p}+\left(2 a_{0}+1\right) C\left(\frac{d}{4 a_{0}}\right)|\nabla u|^{p},
\end{aligned}
$$

where $C(d / 4)$ and $C\left(d / 4 a_{0}\right)$ are the constants given in (2.8). Combining this relation and (2.7), it follows from (2.6) that

$$
\frac{\mathrm{d}}{4} \int_{\mathrm{H}}\left|\nabla \mathrm{u}_{\mathrm{n}}\right|^{p} \mathrm{~d} x \leqslant \int_{\mathrm{H}}\left(\mathrm{C}\left(\frac{\mathrm{d}}{4}\right)+\frac{\mathrm{d}}{4 \mathrm{a}_{0}}\right)|\mathrm{a}(\mathrm{x})|^{\mathrm{p}^{\prime}} \mathrm{d} x+\int_{\mathrm{H}}\left(2 \mathrm{a}_{0}+1\right) \mathrm{C}\left(\frac{\mathrm{d}}{4 \mathrm{a}_{0}}\right)|\nabla \mathrm{u}|^{\mathrm{p}} \mathrm{d} x+\frac{\varepsilon \mathrm{d}}{8} .
$$

Note that $a \in L^{p^{\prime}}\left(\mathbb{R}^{N}\right)$ and $u \in X$. Then there exists $\delta>0$ such that if meas $(H) \leqslant \delta$, then

$$
\int_{H}\left(C\left(\frac{d}{4}\right)+\frac{d}{4 a_{0}}\right)|a(x)|^{p^{\prime}} d x+\int_{H}\left(2 a_{0}+1\right) C\left(\frac{d}{4 a_{0}}\right)|\nabla u|^{p} d x \leqslant \frac{\varepsilon d}{8} .
$$

This together with (2.9) implies that

$$
\frac{\mathrm{d}}{4} \int_{\mathrm{H}}\left|\nabla \mathrm{u}_{\mathrm{n}}\right|^{\mathrm{p}} \mathrm{d} x \leqslant \frac{\varepsilon \mathrm{d}}{8}+\frac{\varepsilon \mathrm{d}}{8}
$$

as claimed. 
On the other hand, there exists a constant $\mathcal{C}>0$ such that

$$
\left|\nabla u_{n}-\nabla u\right|^{p} \leqslant \mathcal{C}\left(\left|\nabla u_{n}\right|^{p}+|\nabla u|^{p}\right),
$$

for all $n \in \mathbb{N}$ and for almost all $x \in \mathbb{R}^{N}$. Since $|\nabla u|^{p} \in L^{1}\left(\mathbb{R}^{N}\right)$ and $\left\{\left|\nabla u_{n}\right|^{p}\right\}$ is uniformly integrable in $\mathbb{R}^{N},\left\{\left|\nabla u_{n}-\nabla u\right|^{p}\right\}$ is also uniformly integrable in $\mathbb{R}^{N}$.

Next, we claim that

$$
\left|\nabla u_{n}(x)-\nabla u(x)\right| \rightarrow 0 \text { as } n \rightarrow \infty,
$$

for almost all $x \in \mathbb{R}^{N}$. From (2.5), it follows that the sequences $\left\{\left(\varphi\left(x, \nabla u_{n}\right)-\varphi(x, \nabla u)\right) \cdot\left(\nabla u_{n}-\nabla u\right)\right\}$ and $\left\{b(x)\left(\left|u_{n}\right|^{p-2} u_{n}-|u|^{p-2} u\right)\left(u_{n}-u\right)\right\}$ converge to 0 in $L^{1}\left(\mathbb{R}^{N} ; \mathbb{R}^{N}\right)$ and $L^{1}\left(\mathbb{R}^{N}\right)$ as $n \rightarrow \infty$, respectively. Hence, there exist a subsequence $\left\{u_{n_{k}}\right\}$ of $\left\{u_{n}\right\}$ and a subset $U$ of $\mathbb{R}^{N}$ of measure 0 such that

$$
\lim _{k \rightarrow \infty}\left(\varphi\left(x, \nabla u_{n_{k}}\right)-\varphi(x, \nabla u)\right) \cdot\left(\nabla u_{n_{k}}-\nabla \mathfrak{u}\right)=0,
$$

for every $x \in \mathbb{R}^{N} \backslash \mathrm{U}$. Let $x \in \mathbb{R}^{N} \backslash \mathrm{U}$. By a similar argument above, we can show that $\left\{\left|\nabla \mathrm{u}_{\mathrm{n}_{\mathrm{k}}}(\mathrm{x})\right|\right\}$ is bounded and so $\left\{\nabla u_{n_{k}}(x)\right\}$ is bounded in $\mathbb{R}^{N}$. By passing to a subsequence, we can assume that $\nabla u_{n_{k}}(x) \rightarrow \xi$ as $k \rightarrow \infty$ for some $\xi \in \mathbb{R}^{N}$. Then we obtain $\varphi\left(x, \nabla u_{n_{k}}\right) \rightarrow \varphi(x, \xi)$ in $\mathbb{R}^{N}$ as $k \rightarrow \infty$ and the relation (2.11) implies that

$$
0=\lim _{k \rightarrow \infty}\left(\varphi\left(x, \nabla u_{n_{k}}\right)-\varphi(x, \nabla \mathfrak{u})\right) \cdot\left(\nabla \mathfrak{u}_{n_{k}}-\nabla \mathfrak{u}\right)=(\varphi(x, \xi)-\varphi(x, \nabla \mathfrak{u})) \cdot(\xi-\nabla \mathfrak{u}) .
$$

It follows from the strict monotonicity of $\varphi$ on $X$ due to (J3) that this relation occurs only if $\xi=\nabla u$, that is, $\nabla u_{n_{k}}(x) \rightarrow \nabla u(x)$ in $\mathbb{R}^{N}$ as $k \rightarrow \infty$. Since these arguments hold for any subsequence of $\left\{\nabla u_{n}\right\}$, we conclude that $\nabla u_{n}(x) \rightarrow \nabla u(x)$ in $\mathbb{R}^{N}$ as $n \rightarrow \infty$ for every $x \in \mathbb{R}^{N} \backslash$ U, i.e., (2.10) holds. Hence, we obtain that $\left\{\left|\nabla u_{n}-\nabla u\right|^{p}\right\}$ converges to zero in $\mathbb{R}^{N}$. Combining this with its uniform integrability, we derive

$$
\lim _{n \rightarrow \infty} \int_{\mathbb{R}^{N}}\left|\nabla u_{n}-\nabla u\right|^{p} \mathrm{~d} x=0
$$

by Vitali's convergence theorem, that is, $\left\|\nabla u_{n}-\nabla u\right\|_{L^{p}\left(\mathbb{R}^{N}\right)} \rightarrow 0$ as $n \rightarrow \infty$. From the compact embedding theorem, we obtain $u_{n} \rightarrow u$ in $L^{p}\left(\mathbb{R}^{N}\right)$ as $n \rightarrow \infty$. It follows from (2.12) that $u_{n} \rightarrow u$ in $W^{1, p}\left(\mathbb{R}^{N}\right)$ as $n \rightarrow \infty$. Therefore, we conclude that $u_{n} \rightarrow u$ in $X$ as $n \rightarrow \infty$, because $\|\cdot\|_{X}$ is equivalent to the norm $\|\cdot\|_{W^{1, p}\left(\mathbb{R}^{N}\right)}$. This completes the proof.

Proceeding arguments analogous to [13, Lemma 3.2], we can see that the functionals $\Psi$ and $\Psi^{\prime}$ are weakly strongly continuous on $\mathrm{X}$. Thus we will omit the proofs here.

Lemma 2.6. Assume that (B) and (F1)-(F2) hold. Then $\Psi$ and $\Psi^{\prime}$ are weakly strongly continuous on $\mathrm{X}$.

With the aid of Lemmas 2.5 and 2.6, we prove that the energy functional $\mathrm{I}_{\lambda}$ satisfies the Cerami condition $\left((C)_{c}\right.$-condition for short $)$, i.e., for $c \in \mathbb{R}$, any sequence $\left\{u_{n}\right\} \subset X$ such that $I_{\lambda}\left(u_{n}\right) \rightarrow c$ and $\left\|I_{\lambda}^{\prime}\left(u_{n}\right)\right\|_{X^{*}}\left(1+\left\|u_{n}\right\|_{X}\right) \rightarrow 0$ as $n \rightarrow \infty$ has a convergent subsequence. This plays a key role in finding a nontrivial weak solution for the given problem.

Remark 2.7. One of the key assumptions for proving that the functional $\mathrm{I}_{\lambda}$ satisfies the $(\mathrm{C})_{\mathrm{c}}$-condition (or Palais-Smale condition) for $c \in \mathbb{R}$ is

$$
f(x, t)=o\left(|t|^{p-1}\right) \text { as }|t| \rightarrow 0 \text {, uniformly for } x \in \mathbb{R}^{N},
$$

see, for instance, $[18,28,29,35]$. However, we prove the following result without the assumption (2.13).

Lemma 2.8. Assume that (B), (J1)-(J5) and (F1)-(F4) hold. Then $\mathrm{I}_{\lambda}$ satisfies the $(\mathrm{C})_{\mathrm{c}}$-condition for any $\lambda>0$. 
Proof. For $c \in \mathbb{R}$, let $\left\{u_{n}\right\} \subset X$ be a $(C)_{c}$-sequence of the functional $I_{\lambda}$, that is,

$$
\mathrm{I}_{\lambda}\left(\mathrm{u}_{\mathrm{n}}\right) \rightarrow \mathrm{c} \quad \text { and }\left\|\mathrm{I}_{\lambda}^{\prime}\left(\mathrm{u}_{\mathrm{n}}\right)\right\|_{\mathrm{X}^{*}}\left(1+\left\|\mathrm{u}_{\mathrm{n}}\right\|_{\mathrm{X}}\right) \rightarrow 0 \quad \text { as } \quad \mathrm{n} \rightarrow \infty,
$$

which shows that

$$
c=I_{\lambda}\left(u_{n}\right)+o(1) \quad \text { and }\left\langle I_{\lambda}^{\prime}\left(u_{n}\right), u_{n}\right\rangle=o(1),
$$

where $\mathrm{o}(1) \rightarrow 0$ as $n \rightarrow \infty$. Since $\mathrm{I}_{\lambda}^{\prime}$ is of type $\left(S_{+}\right)$by Lemmas 2.5, 2.6 and $\mathrm{X}$ is reflexive, it suffices to verify that the sequence $\left\{u_{n}\right\}$ is bounded in $X$.

Assume the contrary that the sequence $\left\{u_{n}\right\}$ is unbounded in $X$. Then we may suppose that $\left\|u_{n}\right\|_{X}>1$ and $\left\|u_{n}\right\|_{X} \rightarrow \infty$ as $n \rightarrow \infty$. Define a sequence $\left\{\ell_{n}\right\}$ by $\ell_{n}=u_{n} /\left\|u_{n}\right\|_{X}$. Then it is obvious that $\left\{\ell_{n}\right\} \subset X$ and $\left\|\ell_{n}\right\|_{X}=1$. Hence, up to a subsequence, still denoted by $\left\{\ell_{n}\right\}$, we obtain $\ell_{n} \rightarrow \ell$ in $X$ as $n \rightarrow \infty$ and according to Lemma 2.1

$$
\ell_{n}(x) \rightarrow \ell(x) \text { a.e. in } \mathbb{R}^{N}, \quad \ell_{n} \rightarrow \ell \text { in } \mathrm{L}^{\mathrm{q}}\left(\mathbb{R}^{\mathrm{N}}\right), \quad \text { and } \quad \ell_{\mathrm{n}} \rightarrow \ell \text { in } \mathrm{L}^{\mathrm{p}}\left(\mathbb{R}^{\mathrm{N}}\right) \quad \text { as } \mathrm{n} \rightarrow \infty .
$$

Set $\Omega_{1}=\left\{x \in \mathbb{R}^{N}: \ell(x) \neq 0\right\}$. Using the assumption (J4) and (2.14), we assert that

$$
\begin{aligned}
c & =I_{\lambda}\left(u_{n}\right)+o(1) \\
& =\int_{\mathbb{R}^{N}} \Phi_{0}\left(x, \nabla u_{n}\right) d x+\frac{1}{p} \int_{\mathbb{R}^{N}} b(x)|u|^{p} d x-\lambda \int_{\mathbb{R}^{N}} F\left(x, u_{n}\right) d x+o(1) \\
& \geqslant \frac{d}{p} \int_{\mathbb{R}^{N}}\left|\nabla u_{n}\right|^{p} d x+\frac{1}{p} \int_{\mathbb{R}^{N}} b(x)|u|^{p} d x-\lambda \int_{\mathbb{R}^{N}} F\left(x, u_{n}\right) d x+o(1) \\
& \geqslant \frac{\min \{d, 1\}}{p}\left\|u_{n}\right\|_{X}^{p}-\lambda \int_{\mathbb{R}^{N}} F\left(x, u_{n}\right) d x+o(1) .
\end{aligned}
$$

Since the sequence $\left\{u_{n}\right\}$ is the $(C)_{c}$-sequence, we get

$$
\int_{\mathbb{R}^{N}} \mathrm{~F}\left(x, u_{n}\right) d x \geqslant \frac{\min \{d, 1\}}{\lambda p}\left\|u_{n}\right\|_{X}^{p}-\frac{c}{\lambda}+\frac{o(1)}{\lambda} \rightarrow \infty \quad \text { as } \quad n \rightarrow \infty .
$$

Moreover, the assumptions (B), (J2) and the Hölder inequality imply that

$$
\begin{aligned}
\mathrm{I}_{\lambda}\left(\mathrm{u}_{n}\right) & =\int_{\mathbb{R}^{N}} \Phi_{0}\left(x, \nabla u_{n}\right) d x+\frac{1}{p} \int_{\mathbb{R}^{N}} \mathrm{~b}(x)|u|^{p} d x-\lambda \int_{\mathbb{R}^{N}} \mathrm{~F}\left(x, u_{n}\right) d x \\
& \leqslant \int_{\mathbb{R}^{N}} \mathrm{a}(x)\left|\nabla u_{n}\right| d x+\frac{a_{0}}{p} \int_{\mathbb{R}^{N}}\left|\nabla u_{n}\right|^{p} d x+\frac{1}{p} \int_{\mathbb{R}^{N}} b(x)|u|^{p} d x-\lambda \int_{\mathbb{R}^{N}} F\left(x, u_{n}\right) d x \\
& \leqslant\|a\|_{L^{p^{\prime}}\left(\mathbb{R}^{N}\right)}\left\|\nabla u_{n}\right\|_{L^{p}\left(\mathbb{R}^{N}\right)}+\left(a_{0}+1\right)\left\|u_{n}\right\|_{X}^{p}-\lambda \int_{\mathbb{R}^{N}} F\left(x, u_{n}\right) d x \\
& \leqslant\left(C_{1}\|a\|_{L^{p^{\prime}}\left(\mathbb{R}^{N}\right)}+a_{0}+1\right)\left\|u_{n}\right\|_{X}^{p}-\lambda \int_{\mathbb{R}^{N}} F\left(x, u_{n}\right) d x,
\end{aligned}
$$

for some constant $C_{1}$. Then we have

$$
\mathrm{C}_{1}\|\mathrm{a}\|_{\mathrm{L}^{p^{\prime}\left(\mathbb{R}^{\mathrm{N}}\right)}}+\mathrm{a}_{0}+1 \geqslant \frac{1}{\left\|\mathrm{u}_{\mathrm{n}}\right\|_{X}^{\mathrm{p}}}\left(\mathrm{I}_{\lambda}\left(\mathrm{u}_{\mathrm{n}}\right)+\lambda \int_{\mathbb{R}^{\mathrm{N}}} \mathrm{F}\left(\mathrm{x}, \mathrm{u}_{\mathrm{n}}\right) \mathrm{dx}\right),
$$

for sufficiently large $n$. The condition (F3) implies that there exists $t_{0}>1$ such that $F(x, t)>|t|^{p}$ for all $x \in \mathbb{R}^{N}$ and $|t|>t_{0}$. Since $F(x, t)$ is continuous on $\mathbb{R}^{N} \times\left[-t_{0}, t_{0}\right]$, there exists a positive constant $M$ such that $|F(x, t)| \leqslant M$, for all $(x, t) \in \mathbb{R}^{N} \times\left[-t_{0}, t_{0}\right]$. Therefore we can choose a real number $M_{0}$ such that $F(x, t) \geqslant M_{0}$, for all $(x, t) \in \mathbb{R}^{N} \times \mathbb{R}$, and thus

$$
\frac{F\left(x, u_{n}(x)\right)-M_{0}}{\left\|u_{n}\right\|_{X}^{p}} \geqslant 0
$$


for all $x \in \mathbb{R}^{N}$ and for all $n \in \mathbb{N}$. By the relation (2.15), we know that $\left|u_{n}(x)\right|=\left|\ell_{n}(x)\right|\left\|u_{n}\right\| x \rightarrow \infty$ as $n \rightarrow \infty$ for all $x \in \Omega_{1}$. Furthermore, it follows from the condition (F3) that

$$
\lim _{n \rightarrow \infty} \frac{F\left(x, u_{n}(x)\right)}{\left\|u_{n}\right\|_{X}^{p}}=\lim _{n \rightarrow \infty} \frac{F\left(x, u_{n}(x)\right)}{\left|u_{n}(x)\right|^{p}}\left|\ell_{n}(x)\right|^{p}=\infty,
$$

for all $x \in \Omega_{1}$. Hence we get that $\left|\Omega_{1}\right|=0$. Indeed, if $\left|\Omega_{1}\right| \neq 0$, then according to (2.16), (2.18), (2.19), (2.20), and the Fatou lemma, we deduce

$$
\begin{aligned}
\frac{1}{\lambda}\left(C_{1}\|a\|_{L^{p^{\prime}\left(\mathbb{R}^{N}\right)}}+a_{0}+1\right) & =\lim _{n \rightarrow \infty} \frac{\left(C_{1}\|a\|_{L^{p^{\prime}}\left(\mathbb{R}^{N}\right)}+a_{0}+1\right) \int_{\mathbb{R}^{N}} F\left(x, u_{n}(x)\right) d x}{I_{\lambda}\left(u_{n}\right)+\lambda \int_{\mathbb{R}^{N}} F\left(x, u_{n}(x)\right) d x} \\
& \geqslant \liminf _{n \rightarrow \infty} \int_{\mathbb{R}^{N}} \frac{F\left(x, u_{n}(x)\right)}{\left\|u_{n}\right\|_{X}^{p}} d x \\
& \geqslant \liminf _{n \rightarrow \infty} \int_{\Omega_{1}} \frac{F\left(x, u_{n}(x)\right)}{\left\|u_{n}\right\|_{X}^{p}} d x-\liminf _{n \rightarrow \infty} \int_{\Omega_{1}} \frac{M_{0}}{\left\|u_{n}\right\|_{X}^{p}} d x \\
& \geqslant \liminf _{n \rightarrow \infty} \int_{\Omega_{1}} \frac{F\left(x, u_{n}(x)\right)-M_{0}}{\left\|u_{n}\right\|_{X}^{p}} d x \\
& \geqslant \int_{\Omega_{1}} \liminf _{n \rightarrow \infty} \frac{F\left(x, u_{n}(x)\right)-M_{0}}{\left\|u_{n}\right\|_{X}^{p}} d x \\
& =\int_{\Omega_{1}} \liminf _{n \rightarrow \infty} \frac{F\left(x, u_{n}(x)\right)}{\left|u_{n}(x)\right|^{p}}\left|\ell_{n}(x)\right|^{p} d x-\int_{\Omega_{1}} \lim _{n \rightarrow \infty} \frac{M_{0}}{\left\|u_{n}\right\|_{X}^{p}} d x \\
& =\infty,
\end{aligned}
$$

which is a contradiction. Thus $\ell(x)=0$ for almost all $x \in \mathbb{R}^{N}$.

Since $I_{\lambda}\left(t u_{n}\right)$ is continuous in $t \in[0,1]$, for each $n \in \mathbb{N}$, there is $t_{n} \in[0,1]$ such that

$$
\mathrm{I}_{\lambda}\left(\mathrm{t}_{\mathrm{n}} \mathrm{u}_{\mathrm{n}}\right):=\max _{\mathrm{t} \in[0,1]} \mathrm{I}_{\lambda}\left(\mathrm{t} \mathrm{u}_{\mathrm{n}}\right) .
$$

Let $\left\{d_{k}\right\}$ be a positive sequence of real numbers such that $\lim _{k \rightarrow \infty} d_{k}=\infty$ and $d_{k}>1$ for any $k$. Then it is clear that $\left\|d_{k} \ell_{n}\right\|_{x}=d_{k}>1$ for any $k$ and $n$. Fix $k$, since $\ell_{n} \rightarrow 0$ strongly in the space $L^{q}\left(\mathbb{R}^{N}\right)$ as $n \rightarrow \infty$, it follows from the continuity of Nemytskii operator that $F\left(x, d_{k} \ell_{n}\right) \rightarrow 0$ in $L^{1}\left(\mathbb{R}^{N}\right)$, which asserts

$$
\lim _{n \rightarrow \infty} \int_{\mathbb{R}^{N}} F\left(x, d_{k} \ell_{n}\right) d x=0 .
$$

Since $\left\|u_{n}\right\|_{X} \rightarrow \infty$ as $n \rightarrow \infty$, we have $\left\|u_{n}\right\|_{X}>d_{k}$ for sufficiently large $n$. Hence we know by (J4) and (2.21) that

$$
\begin{aligned}
& \mathrm{I}_{\lambda}\left(\mathrm{t}_{\mathrm{n}} \mathrm{u}_{\mathrm{n}}\right) \geqslant \mathrm{I}_{\lambda}\left(\frac{\mathrm{d}_{\mathrm{k}}}{\left\|\mathrm{u}_{\mathrm{n}}\right\|_{\mathrm{X}}} \mathrm{u}_{\mathrm{n}}\right)=\mathrm{I}_{\lambda}\left(\mathrm{d}_{\mathrm{k}} \ell_{\mathrm{n}}\right) \\
& =\int_{\mathbb{R}^{N}} \Phi_{0}\left(x, \nabla d_{k} \ell_{n}\right) d x-\lambda \int_{\mathbb{R}^{N}} F\left(x, d_{k} \ell_{n}\right) d x+\frac{1}{p} \int_{\mathbb{R}^{N}} b(x)\left|d_{k} \ell_{n}\right|^{p} d x \\
& \geqslant \frac{d}{p} \int_{\mathbb{R}^{N}} a(x)\left|\nabla d_{k} \ell_{n}\right|^{p} d x-\lambda \int_{\mathbb{R}^{N}} F\left(x, d_{k} \ell_{n}\right) d x+\frac{b_{0}}{p} \int_{\mathbb{R}^{N}}\left|d_{k} \ell_{n}\right|^{p} d x \\
& \geqslant C_{2}\left\|d_{k} \ell_{n}\right\|_{x}^{p}-\lambda \int_{\mathbb{R}^{N}} F\left(x, d_{k} \ell_{n}\right) d x \\
& \geqslant \frac{C_{2}}{2} d_{k}^{p}
\end{aligned}
$$

for sufficiently large $n$, where $C_{2}:=\min \left\{d, b_{0}\right\} / p$. Letting $n$ and $k$ tend to infinity, we conclude that

$$
\lim _{n \rightarrow \infty} I_{\lambda}\left(t_{n} u_{n}\right)=\infty
$$


For any $n$ large enough, this implies that $I_{\lambda}\left(t_{n} u_{n}\right)>0=I_{\lambda}(0)=I_{\lambda}\left(0 u_{n}\right)$, and thus $t_{n}>0$. If $t_{n}<1$, then $\left.\frac{d}{d t} I_{\lambda}\left(t u_{n}\right)\right|_{t=t_{n}}=0$, and so $\left\langle I_{\lambda}^{\prime}\left(t_{n} u_{n}\right), t_{n} u_{n}\right\rangle=0$. If $t_{n}=1$, then we have $\left\langle I_{\lambda}^{\prime}\left(u_{n}\right), u_{n}\right\rangle=0$. Hence we always obtain

$$
\left\langle\mathrm{I}_{\lambda}^{\prime}\left(t_{n} u_{n}\right), t_{n} u_{n}\right\rangle=o(1),
$$

for sufficiently large $n$.

On the other hand, due to the assumptions (J5) and (F4), for all $n$ large enough we obtain that

$$
\begin{aligned}
\frac{1}{\mu} \mathrm{I}_{\lambda}\left(t_{n} u_{n}\right)= & \frac{1}{\mu} \mathrm{I}_{\lambda}\left(t_{n} u_{n}\right)-\frac{1}{\theta \mu}\left\langle I_{\lambda}^{\prime}\left(t_{n} u_{n}\right), t_{n} u_{n}\right\rangle+o(1) \\
= & \frac{1}{\mu} \int_{\mathbb{R}^{N}} \Phi_{0}\left(x, t_{n} \nabla u_{n}\right) d x-\frac{\lambda}{\mu} \int_{\mathbb{R}^{N}} F\left(x, t_{n} u_{n}\right) d x+\frac{1}{p \mu} \int_{\mathbb{R}^{N}} b(x)\left|t_{n} u_{n}\right|^{p} d x \\
& -\frac{1}{\theta \mu} \int_{\mathbb{R}^{N}} \varphi\left(x, t_{n} \nabla u_{n}\right) \cdot\left(t_{n} \nabla u_{n}\right) d x+\frac{\lambda}{\theta \mu} \int_{\mathbb{R}^{N}} f\left(x, t_{n} u_{n}\right) t_{n} u_{n} d x \\
& -\frac{1}{\theta \mu} \int_{\mathbb{R}^{N}} b(x)\left|t_{n} u_{n}\right|^{p} d x+o(1) \\
= & \frac{1}{\theta \mu} \int_{\mathbb{R}^{N}} H\left(x, t_{n} \nabla u_{n}\right) d x+\frac{\lambda}{\theta \mu} \int_{\mathbb{R}^{N}} \mathcal{F}\left(x, t_{n} u_{n}\right) d x+\left(\frac{1}{p \mu}-\frac{1}{\theta \mu}\right) \int_{\mathbb{R}^{N}} b(x)\left|t_{n} u_{n}\right|^{p} d x+o(1) \\
\leqslant & \frac{1}{\theta} \int_{\mathbb{R}^{N}} H\left(x, \nabla u_{n}\right) d x+\frac{\lambda}{\theta} \int_{\mathbb{R}^{N}} \mathcal{F}\left(x, u_{n}\right) d x+\left(\frac{1}{p}-\frac{1}{\theta}\right) \int_{\mathbb{R}^{N}} b(x)\left|u_{n}\right|^{p} d x+o(1) \\
= & \int_{\mathbb{R}^{N}} \Phi_{0}\left(x, \nabla u_{n}\right) d x-\lambda \int_{\mathbb{R}^{N}} F\left(x, u_{n}\right) d x+\frac{1}{p} \int_{\mathbb{R}^{N}} b(x)\left|u_{n}\right|^{p} d x \\
& -\frac{1}{\theta}\left(\int_{\mathbb{R}^{N}} \varphi\left(x, \nabla u_{n}\right) \cdot \nabla u_{n} d x-\lambda \int_{\mathbb{R}^{N}} f\left(x, u_{n}\right) u_{n} d x+\int_{\mathbb{R}^{N}} b(x)\left|u_{n}\right|^{p} d x\right)+o(1) \\
= & I_{\lambda}\left(u_{n}\right)-\frac{1}{\theta}\left\langle I_{\lambda}^{\prime}\left(u_{n}\right), u_{n}\right\rangle+o(1) \rightarrow c \quad a s \quad n \rightarrow \infty,
\end{aligned}
$$

which contradicts (2.22). This completes the proof.

Now, adding the oddity on $f$ and applying the fountain theorem in [34, Theorem 3.6], we shall demonstrate the existence of a sequence of weak solutions for the problem (1.2). To employ the fountain theorem, we consider the following lemma.

Lemma 2.9 ([14]). Let $W$ be a reflexive and separable Banach space. Then there are $\left\{e_{n}\right\} \subseteq W$ and $\left\{f_{n}^{*}\right\} \subseteq W^{*}$ such that

$$
W=\overline{\operatorname{span}\left\{e_{n}: n=1,2, \cdots\right\}}, \quad W^{*}=\overline{\operatorname{span}\left\{\mathbf{f}_{n}^{*}: n=1,2, \cdots\right\}},
$$

and

$$
\left\langle\mathrm{f}_{\mathfrak{i}}^{*}, e_{j}\right\rangle= \begin{cases}1 & \text { if } i=j, \\ 0 & \text { if } i \neq j .\end{cases}
$$

Let us denote $W_{n}=\operatorname{span}\left\{e_{n}\right\}, Y_{k}=\bigoplus_{n=1}^{k} W_{n}$, and $Z_{k}=\overline{\bigoplus_{n=k}^{\infty} W_{n}}$. As a key tool, we recall the following fountain theorem.

Lemma 2.10 ([34]). Let $X$ be a real reflexive Banach space, $I \in C^{1}(X, \mathbb{R})$ satisfies the $(C)_{c}$-condition for any $c>0$ and $\mathrm{I}$ is even. If for each sufficiently large $\mathrm{k} \in \mathbb{N}$, there exist $\rho_{\mathrm{k}}>\delta_{\mathrm{k}}>0$ such that the following conditions hold:

(1) $b_{k}:=\inf \left\{\left(\mathrm{I}(\mathrm{u}): u \in Z_{k},\|u\|_{x}=\delta_{k}\right\} \rightarrow \infty\right.$ as $k \rightarrow \infty$;

(2) $a_{k}:=\max \left\{I(u): u \in Y_{k},\|u\|_{X}=\rho_{k}\right\} \leqslant 0$.

Then the functional I has an unbounded sequence of critical values, i.e., there exists a sequence $\left\{u_{\mathrm{n}}\right\} \subset \mathrm{X}$ such that $\mathrm{I}^{\prime}\left(\mathrm{u}_{\mathrm{n}}\right)=0$ and $\mathrm{I}\left(\mathrm{u}_{\mathrm{n}}\right) \rightarrow \infty$ as $\mathrm{n} \rightarrow \infty$. 
Due to the fountain theorem, we give our main result in this section.

Theorem 2.11. Assume that (B), (J1)-(J5), and (F1)-(F5) hold. If $\Phi_{0}(x,-v)=\Phi_{0}(x, v)$ holds for all $(x, v) \in$ $\mathbb{R}^{\mathrm{N}} \times \mathbb{R}^{\mathrm{N}}$, then for any $\lambda>0$, the problem (1.2) possesses an unbounded sequence of nontrivial weak solutions $\left\{\mathrm{u}_{\mathrm{n}}\right\}$ in $\mathrm{X}$ such that $\mathrm{I}_{\lambda}\left(\mathrm{u}_{\mathrm{n}}\right) \rightarrow \infty$ as $\mathrm{n} \rightarrow \infty$.

Proof. Obviously, $\mathrm{I}_{\lambda}$ is an even functional and satisfies the $(\mathrm{C})_{\mathrm{c}}$-condition. It suffices to show that for every $k \in \mathbb{N}$ there exist $\rho_{k}>\delta_{k}>0$ such that

(1) $b_{k}:=\inf \left\{I_{\lambda}(u): u \in Z_{k},\|u\|_{X}=\delta_{k}\right\} \rightarrow \infty$ as $\quad k \rightarrow \infty$;

(2) $a_{k}:=\max \left\{I_{\lambda}(u): u \in Y_{k},\|u\|_{X}=\rho_{k}\right\} \leqslant 0$.

Set

$$
\alpha_{k}:=\sup _{\mathfrak{u} \in Z_{k},\|\mathfrak{u}\|_{X}=1}\|u\|_{L^{\mathrm{q}}\left(\mathbb{R}^{N}\right)} .
$$

Then we have $\alpha_{k} \rightarrow 0$ as $k \rightarrow \infty$. In fact, assume the contrary, then there exist $\varepsilon_{0}>0, k_{0} \in \mathbb{N}$, and a sequence $\left\{u_{k}\right\}$ in $Z_{k}$ such that

$$
\left\|\mathfrak{u}_{k}\right\|_{X}=1 \text { and }\left\|u_{k}\right\|_{L^{p^{*}}\left(\mathbb{R}^{N}\right)} \geqslant \varepsilon_{0},
$$

for all $k \geqslant k_{0}$. Since the sequence $\left\{u_{k}\right\}$ is bounded in $X$, we may suppose that $u_{k} \rightarrow u$ in $X$ as $k \rightarrow \infty$ for some $u \in X$ and thus

$$
\left\langle\mathrm{f}_{\mathrm{j}}^{*}, \mathfrak{u}\right\rangle=\lim _{\mathrm{k} \rightarrow \infty}\left\langle\mathrm{f}_{\mathrm{j}}^{*}, \mathfrak{u}_{\mathrm{k}}\right\rangle=0,
$$

for $j=1,2, \cdots$. Hence we get $u=0$. However, we observe that

$$
\varepsilon_{0} \leqslant \lim _{k \rightarrow \infty}\left\|u_{k}\right\|_{L^{q}\left(\mathbb{R}^{N}\right)}=\|u\|_{L^{q}\left(\mathbb{R}^{N}\right)}=0,
$$

which provides a contradiction.

For any $u \in Z_{k}$, it follows from the conditions (J4), (F2), the Sobolev inequality, and the Hölder inequality that

$$
\begin{aligned}
\mathrm{I}_{\lambda}(\mathrm{u}) & =\int_{\mathbb{R}^{N}} \Phi_{0}(x, \nabla u) d x+\int_{\mathbb{R}^{N}} \frac{b(x)}{p}|u|^{p} d x-\lambda \int_{\mathbb{R}^{N}} F(x, u) d x \\
& \geqslant \frac{d}{p} \int_{\mathbb{R}^{N}}|\nabla u|^{p} d x+\frac{1}{p} \int_{\mathbb{R}^{N}} b(x)|u|^{p} d x-\lambda \int_{\mathbb{R}^{N}} \frac{|\sigma(x)|}{q}|u|^{q} d x \\
& \geqslant \frac{\min \{d, 1\}}{p}\|u\|_{X}^{p}-\frac{\lambda}{q}\|\sigma\|_{L^{\infty}\left(\mathbb{R}^{N}\right)}\|u\|_{L^{q}\left(\mathbb{R}^{N}\right)}^{q} \\
& \geqslant \frac{\min \{d, 1\}}{p}\|u\|_{X}^{p}-\frac{\lambda C_{3}}{q} \alpha_{k}^{q}\|u\|_{X^{\prime}}^{q}
\end{aligned}
$$

where $C_{3}$ is a positive constant. Choose $\delta_{k}=\left(\lambda C_{3} \alpha_{k}^{q} / \min \{d, 1\}\right)^{1 /(p-q)}$. Since $p<q$ and $\alpha_{k} \rightarrow 0$ as $k \rightarrow \infty$, we assert $\delta_{k} \rightarrow \infty$ as $k \rightarrow \infty$. Hence, if $u \in Z_{k}$ and $\|u\|_{X}=\delta_{k}$, then we deduce that

$$
\mathrm{I}_{\lambda}(\mathrm{u}) \geqslant \min \{\mathrm{d}, 1\}\left(\frac{1}{\mathrm{p}}-\frac{1}{\mathrm{q}}\right) \delta_{\mathrm{k}}^{\mathrm{p}}-\lambda \mathrm{C}_{4} \delta_{\mathrm{k}} \rightarrow \infty \text { as } \mathrm{k} \rightarrow \infty,
$$

for some positive constant $\mathrm{C}_{4}$, which implies (1).

Next, the proof of condition (2) follows the lines of that of [1, Theorem 1.3]. For the sake of convenience, we give the proof. Assume that the condition (2) does not hold for some $k$. Then there exists a sequence $\left\{u_{n}\right\}$ in $Y_{k}$ such that

$$
\left\|u_{n}\right\|_{x} \rightarrow \infty \text { as } n \rightarrow \infty \text { and } \mathrm{I}_{\lambda}\left(\mathrm{u}_{\mathrm{n}}\right) \geqslant 0
$$


Let $\ell_{n}=u_{n} /\left\|u_{n}\right\|_{x}$. Then it is obvious that $\left\|\ell_{n}\right\|_{x}=1$. Since $\operatorname{dim} Y_{k}<\infty$, there exists $\ell \in Y_{k} \backslash\{0\}$ such that up to a subsequence,

$$
\left\|\ell_{\mathrm{n}}-\ell\right\|_{\mathrm{x}} \rightarrow 0 \text { and } \ell_{\mathrm{n}}(\mathrm{x}) \rightarrow \ell(\mathrm{x}) \text {, }
$$

for almost all $x \in \mathbb{R}^{N}$ as $n \rightarrow \infty$. If $\ell(x) \neq 0$, then $\left|u_{n}(x)\right| \rightarrow \infty$ for all $x \in \mathbb{R}^{N}$ as $n \rightarrow \infty$. Hence it follows from the condition (F3) that

$$
\lim _{n \rightarrow \infty} \frac{F\left(x, u_{n}(x)\right)}{\left\|u_{n}\right\|_{x}^{p}}=\lim _{n \rightarrow \infty} \frac{F\left(x, u_{n}(x)\right)}{\left|u_{n}(x)\right|^{p}}\left|\ell_{n}(x)\right|^{p}=\infty,
$$

for all $x \in \Omega_{2}:=\left\{x \in \mathbb{R}^{N}: \ell(x) \neq 0\right\}$. The estimate in Lemma 2.8 implies that

$$
\int_{\Omega_{2}} \frac{\mathrm{F}\left(\mathrm{x}, \mathrm{u}_{\mathrm{n}}(\mathrm{x})\right)}{\left\|\mathrm{u}_{\mathrm{n}}\right\|_{\mathrm{X}}^{\mathrm{p}}} \mathrm{dx} \rightarrow \infty \quad \text { as } \mathrm{n} \rightarrow \infty
$$

Therefore, from the relation (2.17), we have

$$
\begin{aligned}
\mathrm{I}_{\lambda}\left(\mathrm{u}_{\mathrm{n}}\right) & \leqslant\left(\mathrm{C}_{1}\|\mathrm{a}\|_{\mathrm{L}^{p^{\prime}\left(\mathbb{R}^{N}\right)}}+\mathrm{a}_{0}+1\right)\left\|\mathrm{u}_{n}\right\|_{X}^{\mathrm{p}}-\lambda \int_{\Omega_{2}} \mathrm{~F}\left(\mathrm{x}, \mathrm{u}_{\mathrm{n}}\right) \mathrm{d} x \\
& =\left\|\mathrm{u}_{n}\right\|_{X}^{\mathrm{p}}\left(\mathrm{C}_{1}\|\mathrm{a}\|_{\mathrm{L}^{p^{\prime}\left(\mathbb{R}^{N}\right)}}+\mathrm{a}_{0}+1-\lambda \int_{\Omega_{2}} \frac{\mathrm{F}\left(\mathrm{x}, \mathrm{u}_{\mathrm{n}}(\mathrm{x})\right)}{\left\|\mathrm{u}_{\mathrm{n}}\right\|_{X}^{\mathrm{p}}} \mathrm{dx}\right) \rightarrow-\infty \quad \text { as } \mathrm{n} \rightarrow \infty,
\end{aligned}
$$

which contradicts (2.23). This completes the proof.

\section{Existence of a sequence of weak solutions converging to zero}

Now, we deal with the existence of a sequence of weak solutions converging to zero for the problem (1.2). For this, we employ a regularity result (Proposition 3.1). First of all, we need the following additional assumptions for $\varphi, \Phi_{0}$, and $\mathrm{f}$ :

(J6) $\varphi(x, v) \cdot v-p \Phi_{0}(x, v) \geqslant 0$, for all $x \in \mathbb{R}^{N}$ and for all $v \in \mathbb{R}^{N}$.

(F6) There exists a constant $s_{0}>0$ such that $p F(x, t)-f(x, t) t>0$, for all $x \in \mathbb{R}^{N}$ and for $0<|t|<s_{0}$.

(F7) $\lim _{|t| \rightarrow 0} \frac{f(x, t)}{|t|^{p-2} t}=+\infty$ uniformly for all $x \in \mathbb{R}^{N}$.

From the assumptions above, we show the existence of a sequence of solutions for the problem (1.2) converging to zero in the $\mathrm{L}^{\infty}$-norm based on the iteration method in [12, Theorem 4.1], which considered only nonnegative weak solutions. Since the problem (1.2) includes the potential term, more complicated analysis has to be carefully carried out in comparison with nonlinear equations given in [12]. Furthermore, we extend the result to the case of any weak solutions. For a bounded domain, see [20, Theorem 4.1].

Proposition 3.1. Assume that (B), (J1)-(J2), (J4), and (F1)-(F2) hold. If $\mathrm{u}$ is a weak solution of the problem (1.2), then $\mathrm{u} \in \mathrm{L}^{\infty}\left(\mathbb{R}^{\mathrm{N}}\right)$.

Proof. Suppose that $u$ is nonnegative. For a positive constant $M$, define

$$
v_{M}(x)=\min \{u(x), M\}
$$

and choose $v=v_{M}^{k p+1}(k \geqslant 0)$ as a test function in (2.1). Then obviously $v \in X \cap L^{\infty}\left(\mathbb{R}^{N}\right)$ and it follows from (2.1) that

$$
\int_{\mathbb{R}^{N}} \varphi(x, \nabla u) \cdot \nabla v_{M}^{k p+1} d x+\int_{\mathbb{R}^{N}} b(x)|u|^{p-2} u v_{M}^{k p+1} d x=\lambda \int_{\mathbb{R}^{N}} f(x, u) v_{M}^{k p+1} d x .
$$


Due to (J4) and Lemma 2.1, the left-hand side of (3.1) can be estimated as follows:

$$
\begin{aligned}
& \int_{\mathbb{R}^{N}} \varphi\left(x, \nabla v_{M}\right) \cdot \nabla v_{M}^{k p+1} d x+\int_{\mathbb{R}^{N}} b(x)|u|^{p-2} u v_{M}^{k p+1} d x \\
& \geqslant \mathrm{~d}(\mathrm{kp}+1) \int_{\mathbb{R}^{N}} v_{M}^{k p}\left|\nabla v_{M}\right|^{p} \mathrm{~d} x+\int_{\mathbb{R}^{N}} \mathrm{~b}(x) v_{M}^{(k+1) p} \mathrm{~d} x \\
& \geqslant \frac{d(k p+1)}{(k+1)^{p}} \int_{\mathbb{R}^{N}}\left|\nabla v_{M}^{k+1}\right|^{p} d x+\frac{1}{(k+1)^{p}} \int_{\mathbb{R}^{N}} b(x) v_{M}^{(k+1) p} d x \\
& \geqslant \frac{\min \{\mathrm{d}, 1\}}{\mathrm{C}_{5}^{\mathrm{p}}(\mathrm{k}+1)^{\mathrm{p}}}\left(\int_{\mathbb{R}^{N}}\left|v_{M}\right|^{(\mathrm{k}+1) \mathrm{p}^{*}} \mathrm{~d} x\right)^{\frac{\mathrm{p}}{\mathrm{p}^{*}}},
\end{aligned}
$$

for some constant $\mathrm{C}_{5}>0$. By using the assumption (F2) and the Hölder inequality, the right-hand side of (3.1) can be formally estimated from above and we obtain

$$
\begin{aligned}
& \lambda \int_{\mathbb{R}^{N}} f(x, u) v_{M}^{k p+1} d x \leqslant \lambda \int_{\mathbb{R}^{N}}|f(x, u)||u|^{k p+1} d x
\end{aligned}
$$

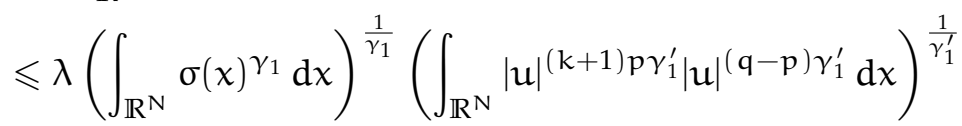

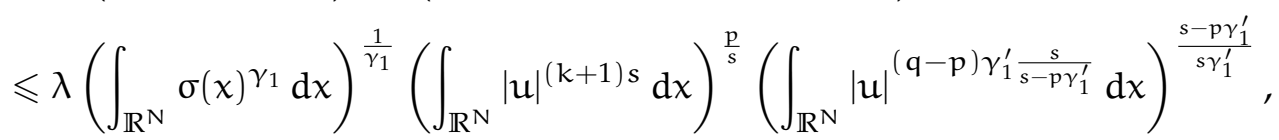

where $s=\frac{p p^{*} \gamma_{1}^{\prime}}{p^{*}-(q-p) \gamma_{1}^{\prime}}$. Obviously $s \leqslant p^{*}, 1<\frac{s}{p \gamma_{1}^{\prime}}$, and $\frac{(q-p) \gamma_{1}^{\prime} s}{s-p \gamma_{1}^{\prime}}=p^{*}$, and hence (3.3) yields

$$
\lambda \int_{\mathbb{R}^{N}} f(x, u) v_{M}^{k p+1} d x \leqslant \lambda\left(\int_{\mathbb{R}^{N}} \sigma(x)^{\gamma_{1}} d x\right)^{\frac{1}{\gamma_{1}}}\left(\int_{\mathbb{R}^{N}}|u|^{p^{*}} d x\right)^{\frac{s-p \gamma_{1}^{\prime}}{s \gamma_{1}^{\prime}}}\left(\int_{\mathbb{R}^{N}}|u|^{(k+1) s} d x\right)^{\frac{p}{s}} .
$$

Now it follows from (3.1), (3.2), (3.4), and the Sobolev inequality that there exists a constant $\mathrm{C}_{6}>0$ (independent of $M$ and $k>0$ ) such that

$$
\left(\int_{\mathbb{R}^{N}}\left|v_{M}\right|^{(k+1) p^{*}} d x\right)^{\frac{p}{p^{*}}} \leqslant C_{6}(k+1)^{p}\left(\int_{\mathbb{R}^{N}}|u|^{(k+1) s} d x\right)^{\frac{p}{s}},
$$

which implies

$$
\left\|v_{M}\right\|_{L^{(k+1) p^{*}\left(\mathbb{R}^{N}\right)}} \leqslant \mathrm{C}_{6}^{\frac{1}{(k+1) p}}(k+1)^{\frac{1}{k+1}}\|u\|_{L^{(k+1) s}\left(\mathbb{R}^{N}\right)}
$$

for any positive constant $M$. The expression (3.5) is a starting point for a bootstrap argument which plays an important role in $L^{\infty}$ - estimates. Since $u \in X$ and hence $u \in L^{p^{*}}\left(\mathbb{R}^{N}\right)$ we can choose $k:=k_{1}$ in (3.5) such that $\left(k_{1}+1\right) s=p^{*}$, i.e., $k_{1}=\frac{p^{*}}{s}-1$. Then we have

$$
\left\|v_{M}\right\|_{L^{\left(k_{1}+1\right) p^{*}}\left(\mathbb{R}^{N}\right)} \leqslant C_{6}^{\overline{\left(k_{1}+1\right) p}}\left(k_{1}+1\right)^{\frac{1}{k_{1}+1}}\|u\|_{L^{\left(k_{1}+1\right) s}\left(\mathbb{R}^{N}\right)}
$$

for any positive constant $M$. Due to $u(x)=\lim _{M \rightarrow \infty} v_{M}(x)$ for almost every $x \in \mathbb{R}^{N}$, the Fatou lemma and (3.6) imply

$$
\|u\|_{L^{\left(k_{1}+1\right) p^{*}}\left(\mathbb{R}^{N}\right)} \leqslant C_{6}^{\frac{1}{\left(k_{1}+1\right) p}}\left(k_{1}+1\right)^{\frac{1}{k_{1}+1}}\|u\|_{L^{\left(k_{1}+1\right) s}\left(\mathbb{R}^{N}\right)} .
$$

Thus, we can choose $k=k_{2}$ in (3.5) such that $\left(k_{2}+1\right) s=\left(k_{1}+1\right) p^{*}=\frac{\left(p^{*}\right)^{2}}{s}$ and repeating the same argument we get

$$
\|u\|_{L^{\left(k_{2}+1\right) p^{*}}\left(\mathbb{R}^{N}\right)} \leqslant C_{6}^{\frac{1}{\left(k_{2}+1\right) p}}\left(k_{2}+1\right)^{\frac{1}{k_{2}+1}}\|u\|_{L^{\left(k_{2}+1\right) s}\left(\mathbb{R}^{N}\right)} .
$$


By induction we obtain

$$
\|u\|_{L^{\left(k_{n}+1\right) p^{*}\left(\mathbb{R}^{N}\right)}} \leqslant C_{6}^{\frac{1}{\left(k_{n}+1\right) p}}\left(k_{n}+1\right)^{\frac{1}{k_{n}+1}}\|u\|_{L^{\left(k_{n}+1\right) s}\left(\mathbb{R}^{N}\right)},
$$

for any $n \in \mathbb{N}$, where $k_{n}+1=\left(\frac{p^{*}}{s}\right)^{n}$. It follows from (3.7) and (3.8) that

$$
\begin{aligned}
\|u\|_{L^{\left(k_{n}+1\right) p^{*}}\left(\mathbb{R}^{N}\right) \leqslant} & C_{6}^{\frac{1}{p} \sum_{j=1}^{n} \frac{1}{k_{j}+1}}\left(k_{1}+1\right)^{\frac{1}{k_{1}+1}}\left(k_{2}+1\right)^{\frac{1}{k_{2}+1}} \\
& \cdots\left(k_{n}+1\right)^{\frac{1}{k_{n}+1}}\|u\|_{L^{\left(k_{1}+1\right) s}\left(\mathbb{R}^{N}\right)}
\end{aligned}
$$

Since $\left(k_{n}+1\right)^{\frac{1}{k_{n}+1}}>1$ and $\lim _{k_{n} \rightarrow \infty}\left(k_{n}+1\right)^{\frac{1}{k_{n}+1}}=1$, there exists $C_{7}>1$ (independent of $k_{n}$ ) such that

$$
\|u\|_{L^{\left(k_{n}+1\right) p^{*}\left(\mathbb{R}^{N}\right)}} \leqslant C_{6}^{\frac{1}{p} \sum_{j=1}^{n} \frac{1}{k_{j}+1}} C_{7}\|u\|_{L^{\left(k_{1}+1\right) s}\left(\mathbb{R}^{N}\right)} .
$$

However $\sum_{j=1}^{n} \frac{1}{k_{j}+1}=\sum_{j=1}^{n}\left(\frac{s}{p^{*}}\right)^{n j}$ and $\frac{s}{p^{*}}<1$. Hence it follows from (3.9) that there exists a constant $\mathrm{C}_{8}>0$ such that

$$
\|u\|_{L^{r n}\left(\mathbb{R}^{N}\right)} \leqslant C_{8}\|u\|_{L^{p^{*}}\left(\mathbb{R}^{N}\right)}
$$

for $r_{n}=\left(k_{n}+1\right) p^{*} \rightarrow \infty$ when $n \rightarrow \infty$. Let us assume that $\|u\|_{L^{\infty}\left(\mathbb{R}^{N}\right)}>C_{8}\|u\|_{L^{p^{*}}\left(\mathbb{R}^{N}\right)}$. Then there exist $\eta>0$ and a set $A$ of positive measure in $\Omega$ such that $u(x) \geqslant C_{8}\|u\|_{L^{p^{*}}\left(\mathbb{R}^{N}\right)}+\eta$ for $x \in A$. It follows that

$$
\begin{aligned}
\liminf _{r_{n} \rightarrow \infty}\left(\int_{\mathbb{R}^{N}}|u(x)|^{r_{n}} d x\right)^{\frac{1}{r_{n}}} & \geqslant \liminf _{r_{n} \rightarrow \infty}\left(\int_{A}|u(x)|^{r_{n}} d x\right)^{\frac{1}{r_{n}}} \\
& \geqslant \liminf _{r_{n} \rightarrow \infty}\left(C_{8}\|u\|_{L^{p^{*}}\left(\mathbb{R}^{N}\right)}+\eta\right)(\operatorname{meas}(A))^{\frac{1}{r_{n}}} \\
& =C_{8}\|u\|_{L^{p^{*}}\left(\mathbb{R}^{N}\right)}+\eta,
\end{aligned}
$$

which contradicts (3.10). Therefore

$$
\|u\|_{L^{\infty}\left(\mathbb{R}^{N}\right)} \leqslant C_{8}\|u\|_{L^{p^{*}}\left(\mathbb{R}^{N}\right)} \leqslant C_{9}
$$

for some constant $\mathrm{C}_{9}>0$.

If $u$ changes sign, we set $u^{+}(x)=\max \{u(x), 0\}$ and $u^{-}(x)=\min \{u(x), 0\}$. Then it is clear that $u^{+} \in X$ and $u^{-} \in X$. Define for each $M>0, v_{M}(x)=\min \left\{u^{+}(x), M\right\}$. Taking again $\varphi=v_{M}^{k p+1}$ as a test function in $X$, we obtain

$$
\int_{\mathbb{R}^{N}} \varphi(x, \nabla u) \cdot \nabla v_{M}^{k p+1} d x+\int_{\mathbb{R}^{N}} b(x)|u|^{p-2} u v_{M}^{k p+1} d x=\lambda \int_{\mathbb{R}^{N}} f(x, u) v_{M}^{k p+1} d x,
$$

which implies that

$$
\int_{\mathbb{R}^{N}} \varphi\left(x, \nabla u^{+}\right) \cdot \nabla v_{M}^{k p+1} d x+\int_{\mathbb{R}^{N}} b(x)\left|u^{+}\right|^{p-2} u^{+} v_{M}^{k p+1} d x=\lambda \int_{\mathbb{R}^{N}} f\left(x, u^{+}\right) v_{M}^{k p+1} d x .
$$

Proceeding a similar argument as above, we obtain $u^{+} \in L^{\infty}\left(\mathbb{R}^{N}\right)$. Likewise, we get $u^{-} \in L^{\infty}\left(\mathbb{R}^{N}\right)$. Therefore $u=u^{+}+u^{-}$is in $L^{\infty}\left(\mathbb{R}^{N}\right)$. This completes the proof.

The following lemma is quoted from [16].

Lemma 3.2 ([16]). Let $\mathrm{I} \in \mathrm{C}^{1}(\mathrm{X}, \mathbb{R})$ where $\mathrm{X}$ is a Banach space. Assume that I satisfies the (PS)-condition, is even and bounded from below, and $\mathrm{I}(0)=0$. If for any $\mathrm{n} \in \mathbb{N}$, there exists an $\mathrm{n}$-dimensional subspace $\mathrm{X}_{\mathrm{n}}$ and $\rho_{\mathrm{n}}>0$ such that

$$
\sup _{X_{n} \cap S_{\rho_{n}}} I<0,
$$

where $S_{\rho}:=\left\{u \in X:\|u\|_{X}=\rho\right\}$, then I has a sequence of critical values $c_{n}<0$ satisfying $c_{n} \rightarrow 0$ as $n \rightarrow \infty$. 
Based on the works of $[9,33]$, we give the following two lemmas.

Lemma 3.3. Assume that (J1)-(J2), (J4), (J6), (F1)-(F2), and (F6) hold. Then

$$
\mathrm{I}_{\lambda}(\mathrm{u})=0=\left\langle\mathrm{I}_{\lambda}^{\prime}(\mathrm{u}), \mathrm{u}\right\rangle \text {, if and only if } \mathrm{u}=0 .
$$

Lemma 3.4. Assume that (F1)-(F2) and (F6)-(F7) hold. Then there exist $\mathrm{t}_{0}>0$ with $\mathrm{t}_{0}<\mathrm{s}_{0} / 2$ and $\tilde{\mathrm{f}} \in \mathrm{C}^{1}\left(\mathbb{R}^{\mathrm{N}} \times\right.$ $\mathbb{R}, \mathbb{R})$ such that $\tilde{f}(x, t)$ is odd in $\mathrm{t}$ and satisfy

$$
\begin{gathered}
\tilde{\mathcal{F}}(x, t):=p \tilde{F}(x, t)-\tilde{f}(x, t) t \geqslant 0, \\
\tilde{\mathcal{F}}(x, t)=0, \quad \text { iff } \quad t \equiv 0 \text { or }|t| \geqslant 2 t_{0},
\end{gathered}
$$

where $\frac{\partial}{\partial \mathrm{t}} \tilde{\mathrm{F}}(\mathrm{x}, \mathrm{t})=\tilde{\mathrm{f}}(\mathrm{x}, \mathrm{t})$.

Proof. Let us define a cut-off function $k \in C^{1}(\mathbb{R}, \mathbb{R})$ satisfying $k(t)=1$ for $|t| \leqslant t_{0}, k(t)=0$ for $|t| \geqslant 2 t_{0}$, $\left|\kappa^{\prime}(t)\right| \leqslant 2 / t_{0}$, and $\kappa^{\prime}(t) t \leqslant 0$. So, we define

$$
\tilde{F}(x, t)=\kappa(t) F(x, t)+(1-\kappa(t)) \xi|t|^{p} \quad \text { and } \quad \tilde{f}(x, t)=\frac{\partial}{\partial t} \tilde{F}(x, t),
$$

where $\xi>0$ is a constant. It is straightforward that

$$
p \tilde{F}(x, t)-\tilde{f}(x, t) t=\kappa(t) \mathcal{F}(x, t)-\kappa^{\prime}(t) t F(x, t)+\kappa^{\prime}(t) t \xi|t|^{p},
$$

where $\mathcal{F}(x, t):=p F(x, t)-f(x, t) t$. For $0 \leqslant|t| \leqslant t_{0}$ and $|t| \geqslant 2 t_{0}$ the conclusion follows. Due to (F7), we choose a sufficiently small $t_{0}>0$ such that $F(x, t) \geqslant \xi t^{p}$ for $t_{0} \leqslant|t| \leqslant 2 t_{0}$. By the assumption $\kappa^{\prime}(t) t \leqslant 0$, we get the conclusion.

Now, we prove the second main result using Proposition 3.1 and Lemmas 3.2 and 3.4.

Theorem 3.5. Assume that (B), (J1)-(J4), (J6), (F1)-(F2), and (F5)-(F7) hold. Moreover, assume that $\Phi_{0}(x,-v)=$ $\Phi_{0}(x, v)$ holds for all $(x, v) \in \mathbb{R}^{N} \times \mathbb{R}^{N}$. Then there exists a positive constant $\lambda^{*}$ such that for every $\lambda \in\left[0, \lambda^{*}\right)$, the problem (1.2) has a sequence of weak solutions $\left\{u_{n}\right\}$ satisfying $\left\|u_{n}\right\|_{L^{\infty}\left(\mathbb{R}^{N}\right)} \rightarrow 0$ as $n \rightarrow \infty$.

Proof. First of all, we will show that $I_{\lambda}$ is coercive on $X$. Let $u \in X$ and $\|u\|_{X}>1$. For the given function $f(x, t)$, we can modify and extend $\tilde{f} \in C^{1}\left(\mathbb{R}^{N} \times \mathbb{R}, \mathbb{R}\right)$ satisfying all properties listed in Lemma 3.4. Define $\tilde{\mathrm{I}}_{\lambda}(\mathrm{u}):=\Phi(\mathrm{u})-\lambda \int_{\mathbb{R}^{N}} \tilde{\mathrm{F}}(\mathrm{x}, \mathrm{u}) \mathrm{d} x$. Then by Lemma 3.4, it is easy to show that $\tilde{\mathrm{I}}_{\lambda} \in \mathrm{C}^{1}(\mathrm{X}, \mathbb{R})$ and is even on X. Moreover, it follows from (F6) and (F7) that for $|u(x)| \leqslant 2 t_{0}$, there exists a positive constant $M_{1}$ such that $M_{1}|u|^{p} \geqslant|F(x, u)|$. Set $\Omega_{3}:=\left\{x \in \mathbb{R}^{N}:|u(x)| \leqslant t_{0}\right\}, \Omega_{4}:=\left\{x \in \mathbb{R}^{N}: t_{0} \leqslant|u(x)| \leqslant 2 t_{0}\right\}$, and $\Omega_{5}:=\left\{x \in \mathbb{R}^{N}: 2 t_{0} \leqslant|u(x)|\right\}$, where $t_{0}$ is given in Lemma 3.4. From (J4), (J6), (3.11), and the conditions of $k$, we have

$$
\begin{aligned}
& \tilde{\mathrm{I}}_{\lambda}(\mathfrak{u}):=\int_{\mathbb{R}^{N}} \Phi_{0}(x, \nabla u) d x+\int_{\mathbb{R}^{N}} \frac{b(x)}{p}|\mathfrak{u}|^{p} \mathrm{~d} x-\lambda \int_{\mathbb{R}^{N}} \tilde{\mathrm{F}}(x, \mathfrak{u}) \mathrm{d} x \\
& \geqslant \frac{\mathrm{d}}{\mathrm{p}} \int_{\mathbb{R}^{N}}|\nabla u|^{\mathrm{p}} \mathrm{d} x+\frac{1}{\mathrm{p}} \int_{\mathbb{R}^{N}} \mathrm{~b}(\mathrm{x})|\mathrm{u}|^{\mathrm{p}} \mathrm{d} x \\
& -\lambda \int_{\Omega_{3}} F(x, u) d x-\lambda \int_{\Omega_{4}}\left\{k(u) F(x, u)+(1-k(u)) \xi|u|^{p}\right\} d x-\lambda \int_{\Omega_{5}} \xi|u|^{p} d x \\
& \geqslant \frac{\min \{d, 1\}}{p}\|u\|_{X}^{p}-\lambda \int_{\Omega_{3}} F(x, u) d x-\lambda \int_{\Omega_{4}} F(x, u) d x-\lambda \int_{\Omega_{4}} \xi|u|^{p} d x-\lambda \int_{\Omega_{5}} \xi|u|^{p} d x \\
& \geqslant \frac{\min \{\mathrm{d}, 1\}}{\mathrm{p}}\|\mathrm{u}\|_{\mathrm{X}}^{\mathrm{p}}-\lambda \int_{\Omega_{3}} \mathrm{M}_{1}|\mathfrak{u}|^{\mathrm{p}} \mathrm{d} x-\lambda \int_{\Omega_{4}} \mathrm{M}_{1}|\mathfrak{u}|^{\mathrm{p}} \mathrm{d} x-\lambda \int_{\Omega_{4}} \xi|\mathfrak{u}|^{\mathrm{p}} \mathrm{d} x-\lambda \int_{\Omega_{5}} \xi|\mathfrak{u}|^{p} \mathrm{~d} x \\
& \geqslant \frac{\min \{\mathrm{d}, 1\}}{\mathrm{p}}\|\mathrm{u}\|_{\mathrm{X}}^{\mathrm{p}}-\lambda\left(\mathrm{M}_{1}+\xi\right) \int_{\mathbb{R}^{\mathrm{N}}}|\mathfrak{u}|^{\mathrm{p}} \mathrm{dx} \\
& \geqslant \frac{\min \{\mathrm{d}, 1\}}{\mathrm{p}}\|\mathrm{u}\|_{\mathrm{X}}^{\mathrm{p}}-\frac{\lambda}{\mathrm{b}_{0}}\left(\mathrm{M}_{1}+\xi\right)\|\mathrm{u}\|_{\mathrm{X}}^{\mathrm{p}} \text {. }
\end{aligned}
$$


If we set

$$
\lambda^{*}:=\frac{\mathrm{b}_{0} \min \{\mathrm{d}, 1\}}{\mathrm{p}\left(\mathrm{M}_{1}+\xi\right)}
$$

then we deduce that for any $\lambda \in\left[0, \lambda^{*}\right), \tilde{\mathrm{I}}_{\lambda}$ is coercive, that is, $\tilde{\mathrm{I}}_{\lambda}(\mathrm{u}) \rightarrow \infty$ as $\|\mathrm{u}\|_{X} \rightarrow \infty$. By a standard argument, $\tilde{\mathrm{I}}_{\lambda}$ satisfies the (PS) $)_{c}$-condition. In order to apply Lemma 3.2, we only need to find for any $n \in \mathbb{N}$, a subspace $X_{n}$ and $\rho_{n}>0$ such that $\sup _{X_{n} \cap S_{\rho_{n}}} \tilde{\tilde{I}}_{\lambda}<0$. For any $n \in \mathbb{N}$ we find $n$ independent smooth functions $\phi_{i}$ for $i=1, \ldots, n$, and define $X_{n}:=\operatorname{span}\left\{\phi_{1}, \ldots, \phi_{n}\right\}$. Due to (J2) and Lemma 3.4, when $\|u\|_{x}<1$ we have that

$$
\begin{aligned}
\tilde{\mathrm{I}}_{\lambda}(\mathrm{u}) & =\int_{\mathbb{R}^{\mathrm{N}}} \Phi_{0}(x, \nabla \mathfrak{u}) \mathrm{d} x+\int_{\mathbb{R}^{\mathrm{N}}} \frac{\mathrm{b}(\mathrm{x})}{\mathrm{p}}|\mathfrak{u}|^{\mathrm{p}} \mathrm{d} x-\lambda \int_{\mathbb{R}^{\mathrm{N}}} \tilde{\mathrm{F}}(\mathrm{x}, \mathrm{u}) \mathrm{d} x \\
& \leqslant\left(\mathrm{C}_{1}\|\mathrm{a}\|_{\mathrm{L}^{p^{\prime}\left(\mathbb{R}^{\mathrm{N}}\right)}}+\mathrm{a}_{0}+1\right)\|\mathrm{u}\|_{\mathrm{X}}^{\mathrm{p}}-\lambda \mathrm{C}_{10} \int_{\mathbb{R}^{\mathrm{N}}} \mathrm{F}(\mathrm{x}, \mathrm{u}) \mathrm{d} \mathrm{x},
\end{aligned}
$$

for a positive constant $C_{10}$. It follows from the assumption (F7) that for a sufficiently large $M_{2}>0$, there exists $\delta_{0}>0$ such that $|t|<\delta_{0}$ implies

$$
\int_{\mathbb{R}^{N}} F(x, t) d x \geqslant \frac{M_{2}}{p} \int_{\mathbb{R}^{N}}|t|^{p} d x
$$

By this relation and the fact that all norms on $X_{n}$ are equivalent, choosing a suitable constant $C_{10}$ and sufficiently small $\rho_{n}>0$, we can obtain

$$
\sup _{X_{n} \cap S_{\rho_{n}}} \tilde{I}_{\lambda}<0 .
$$

By Lemma 3.2, we get a sequence $c_{n}<0$ for $\tilde{\mathrm{I}}_{\lambda}$ satisfying $c_{n} \rightarrow 0$ when $n$ goes to $\infty$. Then for any $u_{n} \in X$ satisfying $\tilde{I}_{\lambda}\left(u_{n}\right)=c_{n}$ and $\tilde{I}_{\lambda}^{\prime}\left(u_{n}\right)=0$, the sequence $\left\{u_{n}\right\}$ is a $(P S)_{0}$-sequence of $\tilde{I}_{\lambda}$ and $\left\{u_{n}\right\}$ has a convergent subsequence. Lemmas 3.3 and 3.4 imply that 0 is the only critical point with 0 energy and the subsequence of $\left\{u_{n}\right\}$ has to converge to 0 . An indirect argument shows the sequence $\left\{u_{n}\right\}$ has to converge to 0 . On the other hand, we have $u_{n} \in C\left(\mathbb{R}^{N}\right)$ due to Proposition 3.1. Since $\left\|u_{n}\right\|_{L^{\infty}\left(\mathbb{R}^{N}\right)} \rightarrow 0$, by Lemma 3.4 again, we deduce $\left\|u_{n}\right\|_{C\left(\mathbb{R}^{N}\right)} \leqslant t_{0}$. Thus $\left\{u_{n}\right\}$ are weak solutions of the problem (1.2). The proof is complete.

\section{Acknowledgment}

Y.-H. Kim was supported by the National Research Foundation of Korea Grant funded by the Korean Government (NRF-2016R1D1A1B03935866). J. Lee was supported by Basic Science Research Program through the National Research Foundation of Korea (NRF) funded by the Ministry of Education (20090093827).

\section{References}

[1] C. O. Alves, S.-B. Liu, On superlinear $p(x)$-Laplacian equations in $\mathbf{R}^{\mathbf{N}}$, Nonlinear Anal., 73 (2010), 2566-2579. 1, 1, 2

[2] A. Ambrosetti, P. H. Rabinowitz, Dual variational methods in critical point theory and applications, J. Functional Analysis, 14 (1973), 349-381. 1

[3] T. Bartsch, Infinitely many solutions of a symmetric Dirichlet problem, Nonlinear Anal., 20 (1993), 1205-1216. 1

[4] T. Bartsch, Topological methods for variational problems with symmetries, Lecture Notes in Mathematics, SpringerVerlag, Berlin, (1993). 1

[5] T. Bartsch, Z. Q. Wang, Existence and multiplicity results for some superlinear elliptic problems on $\mathbf{R}^{\mathrm{N}}$, Comm. Partial Differential Equations, 20 (1995), 1725-1741. 1

[6] G. Bin, On superlinear $\mathrm{p}(\mathrm{x})$-Laplacian-like problem without Ambrosetti and Rabinowitz condition, Bull. Korean Math. Soc., 51 (2014), 409-421. 2.4

[7] Y. Chen, X. H. Tang, Ground state solutions for p-superlinear p-Laplacian equations, J. Aust. Math. Soc., 97 (2014), 48-62. 1 
[8] E. B. Choi, Y.-H. Kim, Three solutions for equations involving nonhomogeneous operators of p-Laplace type in $\mathbb{R}^{\mathrm{N}}$, J. Inequal. Appl., 2014 (2014), 15 pages. 1, 2

[9] E. B. Choi, J.-M. Kim, Y.-H. Kim, Infinitely many solutions for equations of $\mathrm{p}(\mathrm{x})$-Laplace type with the nonlinear Neumann boundary condition, Proc. Roy. Soc. Edinburgh Sect. A, (in press). 1, 3

[10] F. Colasuonno, P. Pucci, C. Varga, Multiple solutions for an eigenvalue problem involving p-Laplacian type operators, Nonlinear Anal., 75 (2012), 4496-4512. 1, 2

[11] P. De Nápoli, M. C. Mariani, Mountain pass solutions to equations of p-Laplacian type, Nonlinear Anal., 54 (2003), 1205-1219. 1

[12] P. Drábek, A. Kufner, F. Nicolosi, Quasilinear elliptic equations with degenerations and singularities, De Gruyter Series in Nonlinear Analysis and Applications, Walter de Gruyter \& Co., Berlin, (1997). 1, 3

[13] X.-L. Fan, X.-Y. Han, Existence and multiplicity of solutions for $\mathrm{p}(\mathrm{x})$-Laplacian equations in $\mathbb{R}^{\mathrm{N}}$, Nonlinear Anal., 59 (2004), 173-188. 2

[14] X.-L. Fan, Q.-H. Zhang, Existence of solutions for $\mathrm{p}(\mathrm{x})$-Laplacian Dirichlet problem, Nonlinear Anal., 52 (2003), 18431852. 2.9

[15] Z.-J. Guo, Elliptic equations with indefinite concave nonlinearities near the origin, J. Math. Anal. Appl., 367 (2010), 273-277. 1

[16] H. P. Heinz, Free Ljusternik-Schnirelman theory and the bifurcation diagrams of certain singular nonlinear problems, J. Differential Equations, 66 (1987), 263-300. 3, 3.2

[17] L. Jeanjean, On the existence of bounded Palais-Smale sequences and application to a Landesman-Lazer-type problem set on $\mathbf{R}^{\mathrm{N}}$, Proc. Roy. Soc. Edinburgh Sect. A, 129 (1999), 787-809. 1

[18] C. Ji, On the superlinear problem involving the $p(x)$-Laplacian, Electron. J. Qual. Theory Differ. Equ., 2011 (2011), 9 pages. $1,2.7$

[19] Y.-T. Jing, Z.-L. Liu, Infinitely many solutions of p-sublinear p-Laplacian equations, J. Math. Anal. Appl., 429 (2015), 1240-1257. 1

[20] A. Lê, Eigenvalue problems for the p-Laplacian, Nonlinear Anal., 64 (2006), 1057-1099. 3

[21] V. K. Le, On a sub-supersolution method for variational inequalities with Leray-Lions operators in variable exponent spaces, Nonlinear Anal., 71 (2009), 3305-3321. 2

[22] J. S. Lee, Y.-H. Kim, Existence and multiplicity of solutions for nonlinear elliptic equations of p-Laplace type in $\mathbb{R}^{\mathrm{N}}$, Filomat, 30 (2016), 2029-2043. 1, 1

[23] J. Lee, Y.-H. Kim, Multiplicity results for nonlinear Neumann boundary value problems involving p-Laplace type operators, Bound. Value Probl., 2016 (2016), 25 pages. 1

[24] S. D. Lee, K.-S. Park, Y.-H. Kim, Existence and multiplicity of solutions for equations involving nonhomogeneous operators of $\mathrm{p}(\mathrm{x})$-Laplace type in $\mathbb{R}^{\mathrm{N}}$, Bound. Value Probl., 2014 (2014), 17 pages. 1, 2, 2

[25] G.-B. Li, C.-Y. Yang, The existence of a nontrivial solution to a nonlinear elliptic boundary value problem of p-Laplacian type without the Ambrosetti-Rabinowitz condition, Nonlinear Anal., 72 (2010), 4602-4613. 1

[26] X.-Y. Lin, X. H. Tang, Existence of infinitely many solutions for p-Laplacian equations in $\mathbb{R}^{\mathrm{N}}$, Nonlinear Anal., 92 (2013), 72-81. 1

[27] S.-B. Liu, On ground states of superlinear p-Laplacian equations in R N , J. Math. Anal. Appl., 361 (2010), 48-58. 1

[28] S.-B. Liu, S. J. Li, Infinitely many solutions for a superlinear elliptic equation, (Chinese) Acta Math. Sinica (Chin. Ser.), 46 (2003), 625-630. 1, 2.7

[29] O. H. Miyagaki, M. A. S. Souto, Superlinear problems without Ambrosetti and Rabinowitz growth condition, J. Differential Equations, 245 (2008), 3628-3638. 1, 2.7

[30] D. Naimen, Existence of infinitely many solutions for nonlinear Neumann problems with indefinite coefficients, Electron. J. Differential Equations, 2014 (2014), 12 pages. 1

[31] Z. Tan, F. Fang, On superlinear p (x)-Laplacian problems without Ambrosetti and Rabinowitz condition, Nonlinear Anal., 75 (2012), 3902-3915. 1

[32] C. E. Torres Ledesma, Existence and symmetry result for fractional p-Laplacian in $\mathbb{R}^{n}$, Commun. Pure Appl. Anal., 16 (2017), 99-113. 1

[33] Z.-Q. Wang, Nonlinear boundary value problems with concave nonlinearities near the origin, NoDEA Nonlinear Differential Equations Appl., 8 (2001), 15-33. 1, 3

[34] M. Willem, Minimax theorems, Progress in Nonlinear Differential Equations and their Applications, Birkhäuser Boston, Inc., Boston, MA, (1996). 1, 1, 2, 2, 2.10

[35] L. S. Yu, Nonlinear p-Laplacian problems on unbounded domains, Proc. Amer. Math. Soc., 115 (1992), 1037-1045. 2.7

[36] W.-M. Zou, Variant fountain theorems and their applications, Manuscripta Math., 104 (2001), 343-358. 1 